\title{
Wetland water quality assessment in cold and dry regions (Case study: Choghakhor wetland, Iran)
}

\author{
Eisa Ebrahimi Dorche*, Pejman Fathi, Alireza Esmaeili Ofogh \\ Division of Fisheries, Department of Natural Resources, Isfahan University of Technology, Isfahan 8415683111, Iran, \\ e-mail: e_ebrahimi@cc.iut.ac.ir ( ${ }^{\star}$ corresponding author)
}

\begin{abstract}
Wetlands are among the most productive ecosystems in the world. They provide numerous beneficial services for people and wildlife. The most important services are improving water quality and wildlife habitats. The complex, dynamic relationships between the organisms inhabiting the wetland environment are called food webs. Both water quality and high levels of nutrients are crucial for improvement of the food web. Many bird species rely on wetlands for food, water and shelter, especially during migration and breeding. The water quality of Choghakhor Wetland was evaluated from April 2010 to March 2011, by measuring some physicochemical parameters and doing benthic macroinvertebrate analysis. Sampling was done in 3 replicates with alternation of 45 days. The resolute was divided into more than 25 identified macroinvertebrate families which belonged to 5 classes and 12 orders. The correlation between biological indices and water quality parameters showed that bioindicators and community indices could be used for the evaluation of water quality in this wetland. The water quality of Choghakhor Wetland was classified as average or in the severe pollution class, according to these indices. It can be concluded that bird migration is at risk due to the decline in the overall health of the Choghakhor Wetland ecosystem.
\end{abstract}

Key words: benthic bacroinvertebrate, bioindicators, water quality, Choghakhor Wetland, Iran

\section{Introduction}

Wetlands are areas of marsh, ponds and swamps, whether natural or artificial, permanent or temporary, with water that is static or flowing, fresh, brackish or salty, including areas of marine water, the depth of which at low tide does not exceed six meters (Getachew et al. 2018). Wetland ecosystems are among the most productive environments on the planet, sustaining more life than many tropical forests (Mitsch and Gosselink 2007). These resources have immense ecological importance and provide habitats for numerous endemic plant and animal species (Hettiarchchi et al. 2011). Globally, freshwater wetlands, which cover approximately $1 \%$ of the earth's surface, support over $40 \%$ of the world's plant species and $12 \%$ of all animal species (RCW 2002). Migratory birds, which migrate for long periods need high energy and safe environment to survive the cold winter, so they choose areas in which they can find better food and spawning places. Wetlands serve as stopover sites for thousands of migratory bird species including some worldwide endangered ones (Yimer and Mengistou 2009).

The geographical location of Iran, its seasonal climate and the fact that it lies beneath two main cor- ridors for bird migration make Iran's wetlands vital for supporting natural bird migration patterns, a phenomenon that plays a determinative role in attracting tourists, economics and the health of the community. Non-migratory birds and migratory ones can be considered as an indicator for ecosystem health status. More than eighty species of birds, (including geese, cranes, swans, ducks, scaup, sandpipers and birds of prey) (Mansoori 2008) and about ten species of mammals in Iran are closely related to the wetlands for spawning and a nursery environment, resting place and also as shelter for protection against predators (Harrington et al. 1977). Choghakhor Wetland is one of the most important wetlands in Iran due to its ecological value, exquisite landscapes, plant and aquatic animal diversity as well as its attractiveness for ecotourism. In terms of bird criteria, this wetland has 3 species with a low population, 2 species in danger of extinction (white-headed and marbled ducks), a population of more than 25 thousand birds per year, 3 oviparous species which include $1 \%$ of the population, and bird diversity is considered to be more than 20 important species (Mansoori 2008).

The main reasons for wetland loss and degradation are human activities and climate change (Mereta et 
al. 2013). Wetlands dry out, and the use of pesticides which eventually enter the wetlands together with excessive hunting are the major threats to bird life. Water quality assessment in wetlands is essential to conserve the diversity of aquatic organisms. Macroinvertebrates have been identified as excellent indicators of water quality as they respond rapidly to environmental changes. Their abundance, diversity and short life cycle make them ideal subjects for the assessment of the ecological condition of wetlands (Rader et al. 2001). They are the most abundant group of organisms in freshwater wetlands and play an important role in the overall functionality of these unique ecosystems as they occupy a central position in wetland food chains (Batzer et al. 1999). Macroinvertebrate monitoring is, thus, frequently used in environmental quality assessment of aquatic ecosystems (Cabecinha et al. 2007; Pinel-Alloul et al. 1995).

Biological indicators are numerical values by which one can combine a quantitative amount of species diversity with qualitative information about the ecological sensitivity of each taxon among others (Czeniawska-Kusza 2005). Indicators have been developed for specific geographic areas, such as Belgium bio-indicators (BBI) or the BMWP points system, which has been prepared to monitor water quality in England. They have also been adopted in some other countries successfully (Czeniawska-Kusza 2005; Azrina et al. 2005; Nemati et al. 2009). Burton et al (1999) used the Biological Health Index (IBI), which was based on benthic invertebrates in Lake Huron coastal lagoons. Helgen (2002) used the BIB index, based on macroinvertebrates, for wetland degradation in Minnesota. Baty et al (2005) used Biological indicators BMWP, ASPT, Simpson's diversity index and physicochemical parameters for potential ecological assessments of House and whittle wetlands. Galbrand et al. (2007) assessed a protected wetland in an industrial park biologically using BMWP, ASPT, ETSD and Myflie abundance macroinvertebrates. Taowu et al (2008) evaluated the quality of Lake Taiha water using the HBI index, richness and taxonomic position. A similar study was performed on Asan wetland in India and macroinvertebrates were used as bio-indicators for the health assessment of the wetland (Ramesh and Jitendra 2009). However, biological assessments of rivers and lakes in Iran are very limited. The study of macroinvertebrates as an impact indicator can reveal the occurrence of intermittent or unrecorded chemical pollution incidents (Rosenberg and Resh 1993; Fishar and Williams 2008). Therefore, the present study was an attempt to assess the water quality of the Choghakhor Wetland using benthic communities and physicochemical factors.

\section{Study area}

The study area was Choghakhor wetland covering approximately 2300 hectares together with an area in which hunting is forbidden comprising 2500 hectares. Water depth of the wetland reaches 3 meters in March at the peak condition and its volume is 40 million $\mathrm{m}^{3}$. It is located between the Baraftab mountains in the north and Kelar in the southern part of the GandomanBoldaji Plain that is located in Chaharmahal Bakhtiari Province. Wetland water resources are mainly provided through atmospheric precipitation and springs in the Kelar mountain ridge. The extent of this area is 768 $\mathrm{km}^{2}$ of which $222 \mathrm{~km}^{2}$ form the plain. The GandomanBoldaji Plain is located between $31^{\circ} 50^{\prime}$ and $32^{\circ} 00^{\prime}$ north and $51^{\circ} 00^{\prime}$ and $51^{\circ} 10^{\prime}$ east (Fig. 1). The average annual rainfall is $380 \mathrm{~mm}$. Due to the calcareous water, ground water is relatively desirable (Shivandi et al. 1999). Among the birds of the wetland can be mentioned all kinds of goose and duck, redshank, ruddy shelduck, white stork, coot, pelican, grebe, cormorant, flamingo, birds of prey, ibis, swans, plovers, seagulls and waders. The following wetland fish can be found: Aphanius spp., Barbel spp., and introduced fish in the wetland are common carp (Cyprinus carpio), silver carp (Hypophthalmichthys molitrix), grass carp (Ctenopharyngodon idella) and narrow-barred mackerel. The unique feature of the Choghakhor wetland is the presence of some fishs belonging to the cyprinodontidae family. The wetland vegetation is composed of marginal, hydrophilic, submerged and free floating species such as willow, couchgrass, potamogeton, polygonum. There are 29 plant species in this wetland, which include $56 \%$ emergent, $41 \%$ submerged and 3\% free floating plants (Mansoori 2008). Photography, bird watching, fishing and boating are the wetland's recreational activities.

\section{Materials and methods}

The sampling was performed in eight stages with a time interval of 45 days in four seasons, from April 2010 to March 2011 (25 April, 10 June, 27 July, 11 September, 27 October, 11 December, 25 January, 11 March). Ten sampling stations were considered with a distance of $1 \mathrm{~km}$ between adjacent stations. By using a topographic map and the lattice method, these locations were determined on the map. The intersections of grid lines were selected as sampling stations (Table 1). A GPS device was used to find the sampling stations (Tiner 1999).

In order to analyze the physicochemical parameters of water at each station, one liter of water was taken from a depth of $30 \mathrm{~cm}$ and transported to the laboratory in standard conditions. Physicochemical parameters of water were analyzed using the standard method 


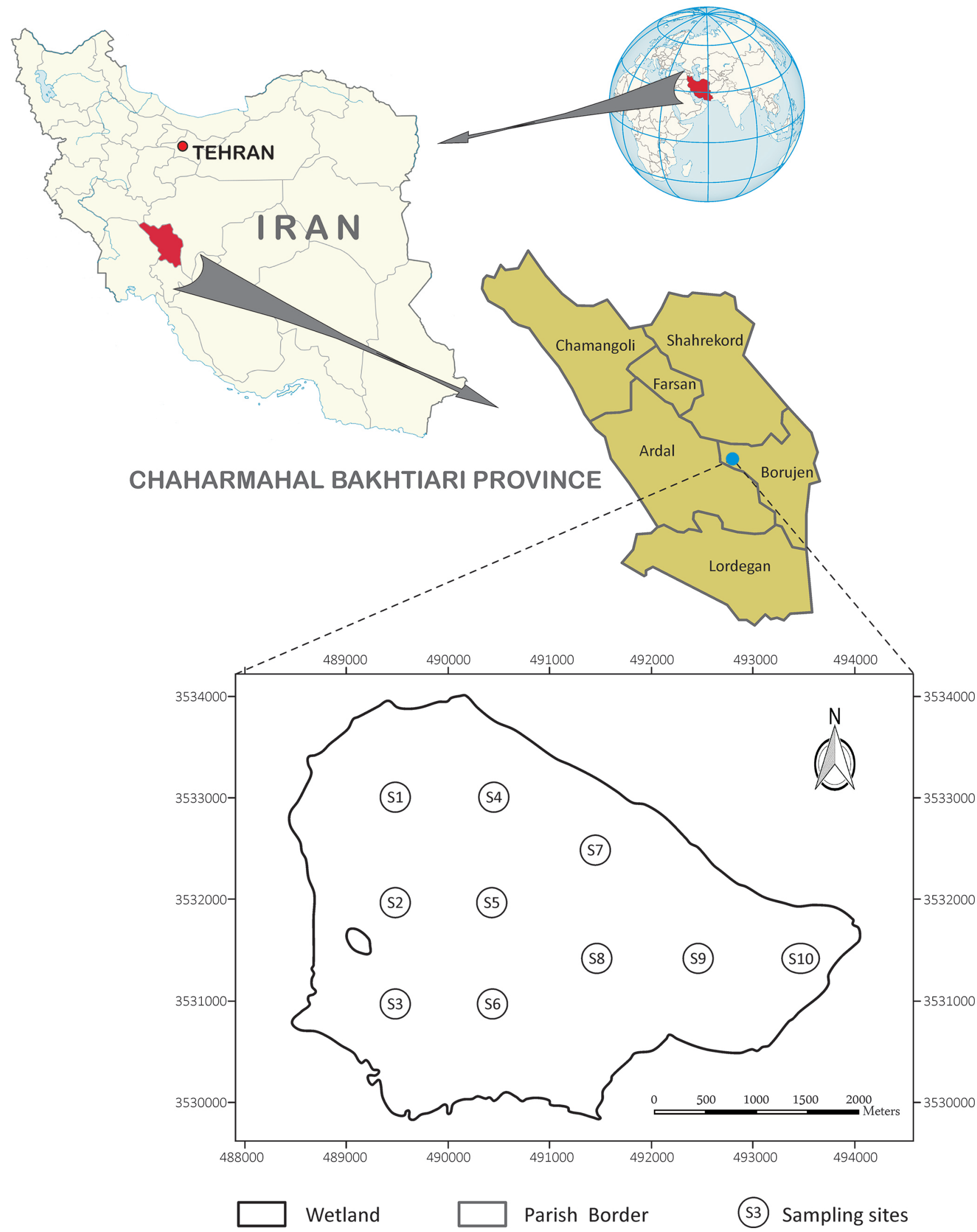

Fig. 1. Map of Choghakhor Wetland and Chaharmahal Bakhtiari with an indication of the study area 
Table 1. Geolocation of the studied stations in Choghakhor Wetland

\begin{tabular}{|c|c|c|}
\hline Station No. & \multicolumn{2}{|c|}{ UTM coordinates } \\
\hline 1 & $\mathrm{X}=489500$ & $\mathrm{Y}=3533000$ \\
\hline 2 & $\mathrm{X}=489500$ & $\mathrm{Y}=3532000$ \\
\hline 3 & $\mathrm{X}=489500$ & $\mathrm{Y}=3531000$ \\
\hline 4 & $\mathrm{X}=490500$ & $\mathrm{Y}=3533000$ \\
\hline 5 & $\mathrm{X}=490500$ & $\mathrm{Y}=3532000$ \\
\hline 6 & $\mathrm{X}=490500$ & $\mathrm{Y}=3531000$ \\
\hline 7 & $\mathrm{X}=491500$ & $\mathrm{Y}=3532500$ \\
\hline 8 & $\mathrm{X}=491500$ & $\mathrm{Y}=3531500$ \\
\hline 9 & $\mathrm{X}=492500$ & $\mathrm{Y}=3531500$ \\
\hline 10 & $\mathrm{X}=493500$ & $\mathrm{Y}=3531500$ \\
\hline
\end{tabular}

Table 2. Evaluation of water quality using the Shannon-Wiener diversity index according to Wilhm and Dorris (1968)

\begin{tabular}{|c|c|}
\hline Shannon-Wiener Diversity Index & Water quality \\
\hline $3-5$ & Clean waters \\
\hline $1-3$ & Moderately polluted waters \\
\hline$<1$ & Severely polluted waters \\
\hline
\end{tabular}

APHA (APHA, 1992). Eighteen parameters, including temperature, depth, dissolved oxygen, nitrate, nitrite, phosphate, ammonium, alkalinity, hardness, turbidity, electrical conductivity, chlorophyll- $a$, pH, TDS (Total dissolved solids), TSS (Total suspended solids), TS (Total solids), $\mathrm{BOD}_{5}$ (Biochemical oxygen demand) and COD (Chemical oxygen demand) were selected. The values used for each parameter was the mean of three replicates for each station in each stage. Sampling of sediment was done using an Ekman grab with a surface area of $400 \mathrm{~cm}^{2}$ in all stations with three replicates. The content of each sample was washed in the field using a sieve with a mesh size of $0.50 \mathrm{~mm}$. All captured organisms were placed in plastic bottles and preserved in $4 \%$ formalin. Benthic macroinvertebrate identification was carried out to the lowest possible taxonomic level in the laboratory based on keys (Hynes 1977; Milligan 1997; Pescador et al. 2004). Benthic macro-invertebrates in each sample were counted and identified to the family level. Taxa richness (number of families identified in each station), Margalef's index (Washington 1984), Simpson's diversity index (Washington 1984; Krebs 2001) and the Shannon-Wiener diversity index (Washington 1984) were summarized. Also, the biological indices HBI, BMWP and ASPT were calculated.
Table 4. Classification of water quality based on the Biological Monitoring Working Party (Mandaville 2002)

\begin{tabular}{|c|c|l|}
\hline Score & Water quality & \multicolumn{1}{c|}{ Meaning } \\
\hline $0-10$ & Very critical & Strongly polluted waters \\
\hline $11-40$ & Dubious & Polluted waters (altered system) \\
\hline $41-70$ & Passable & Evidences of mild pollution effects \\
\hline $71-100$ & Good & Clean but system a little altered \\
\hline$>100$ & Very good & $\begin{array}{l}\text { Non polluted or not sensibly altered } \\
\text { system }\end{array}$ \\
\hline
\end{tabular}

The following equations were used for calculating these indices:

- Margalef's richness index $(R)$ :

$$
R=(S-1) / \ln N,
$$

where $S$ is the total number of taxa, and $N$ is the total number of specimens.

- Simpson's diversity index $(D)$ :

$$
D=1-\Sigma\left(p_{i}\right)^{2},
$$

where $p_{i}$ is the proportion of individuals of species " $i$ ".

- Shannon-Wiener diversity index $(H)$ :

$$
\mathrm{H}=-\sum\left(p_{i} \ln p_{i}\right)
$$

where $p_{i}$ is the proportion of individuals of species " $i$ ".

The Shannon-Wiener diversity index was applied and for quality assessment it was compared with the values in Table 2.

Hilsenhoff's biotic index ( $H B I)$, based on the following equation (Barbour et al. 1999) and tables index scores (Hilsenhoff 1988; Bode et al. 1996), were calculated and for quality assessment, it then compared with the values in Table 3 . The biotic index formulation is as follows:

$$
\mathrm{HBI}=\sum\left(n_{i} \times t_{i}\right) / N \text {, }
$$

where $n_{i}$ is the number of individuals within a taxon " $i$ ", $t$. is a tolerance value of taxon " $i$ ", and $N$ is the total number of specimens in each sample.

The BMWP index at each station and stage was calculated by adding the individual scores of the families

Table 3. Evaluation criteria of water quality using the family-level biotic index (Hilsenhoff 1988; Walen 2002)

\begin{tabular}{|c|c|l|}
\hline Family Biotic Index & Water quality & \multicolumn{1}{|c|}{ Degree of organic pollution } \\
\hline $0.00-3.50$ & Excellent & No apparent organic pollution \\
\hline $3.51-4.50$ & Very good & Possible slight organic pollution \\
\hline $4.51-5.50$ & Good & Some organic pollution \\
\hline $5.51-6.50$ & Fair & Fairly significant organic pollution \\
\hline $6.51-7.50$ & Fairly Poor & Significant organic pollution \\
\hline $7.51-8.50$ & Poor & Very significant organic pollution \\
\hline $8.51-10.00$ & Very Poor & Severe organic pollution \\
\hline
\end{tabular}


Table 5. Classification of water quality based on the average score per taxon (Mandaville 2002)

\begin{tabular}{|c|c|}
\hline ASPT value & Water quality assessment \\
\hline$>6$ & Clean Water \\
\hline $5-6$ & Doubtful quality \\
\hline $4-5$ & Probable moderate pollution \\
\hline$>4$ & Probable severe pollution \\
\hline
\end{tabular}

and comparing it with the score value in Table 4. (Wally and Hawkes 1996; Wally and Hawkes 1997). The ASPT index was calculated by the ratio of BMWP values to the number of families, respectively (Wally and Hawkes 1996; Wally and Hawkes 1997). The values obtained were compared with the ASPT values in Table 5.

\section{Analytical procedures}

The data analysis was performed using SPSS 18 software. Normalization and homogenization of variances of data were investigated using the Kolmogorov - Smirnov and Leven tests. In order to evaluate the differences between sampling stations and stages, one-way ANOVA analysis and Duncan test were performed. To investigate the association between biological indicators (BMWP, ASPT and HBI) and indices of diversity and richness, the normal distribution of the Pearson correlation coefficient was used (Zar 1999). To show spatial and temporal variations of data, Box and Whisker plot diagrams were drawn using Statgraphics software. In these charts statistics such as mean, median, $50 \%$ of the average data, outlier values, extreme values as well as the range of data are shown after deleting the outliers and extreme values (Fig. 2). Here, data is shown as outliers values, which are specified in the range of more than 1.5 to less than 3 times the standard deviation from the average. Extreme values are also referred to as data that are more than 3 times the standard deviation of the mean. A cluster analysis was also performed using bray curtis distance and single linkage.

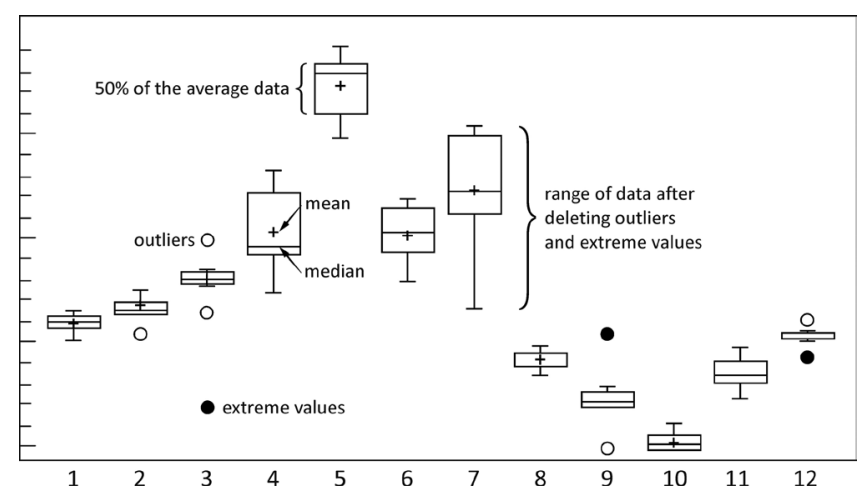

Fig. 2. An example of box and whisker plot diagram

\section{Results and discussion}

\section{Physicochemical factors}

Mean and standard deviation of the measured physicochemical characters in various stations of sampling are shown in Table 6.

Mean and standard deviation of the measured physicochemical characters in various stages of sampling are shown in Table 7.

Water quality characters in sampling stations (except in some cases) did not show significant differences (Table 6), but in different stages of sampling they were significantly different at the level of $1 \%$ (Table 7). The highest and lowest temperature was observed in stages 4 (late summer) and 7 (early winter), respectively. Temperatures in aquatic ecosystems are closely linked to air temperature (Desta et al 2012) Thus, it is clear that an increase in air temperature in summer should be followed by similar increases in water temperature.

Depth of Water of the wetland showed a decreasing trend from early spring (April) to early fall (October) due to the use of water for agriculture and the reduction of seasonal rainfalls. During the cold seasons, with the increase of rainfalls and reduction of water usage, the water depth increased from late autumn (December) to late winter (March). The amount of oxygen was decreased possibly by the reduction of oxygen solubility from April to July. Inclination of oxygen solubility could be due to rising water temperature, an increase in the activity of organisms and the oxidation process of organic matter. Then, because of the intensity of photosynthesis by the aquatic plants the oxygen concentration of water had an upward trend from September to December, contrary to the increase of water temperature and oxidation of organic matter. The fall of oxygen levels in winter was due to the decline in the growth of aquatic plants. In general, we can say that there were two peaks in spring and autumn. Dissolved oxygen levels were high $\left(>7 \mathrm{mg} \mathrm{dm}^{-3}\right)$ at all stations and stages and were under the influence of photosynthesis (Li et al. 2009), and according to USEPA, were considered to be favorable for aquatic life. Dissolved Oxygen (DO) is an indicator of a water body's ability to support aquatic life; hence, it is essential for good water quality. Its amount is directly related to the population size and community of aerobic bacteria that an aquatic system can support (Yimer and Mengistou 2009). Also, Ramesh and Jitendra (2009) have confirmed a positive correlation between the vegetation and the amount of dissolved oxygen in Asan wetland.

Water transparency decreased from April to October owing to the accelerated growth of vegetation which reduced Secchi disk visibility and clarity from December to March. Yimer and Mengistou 2009 found 


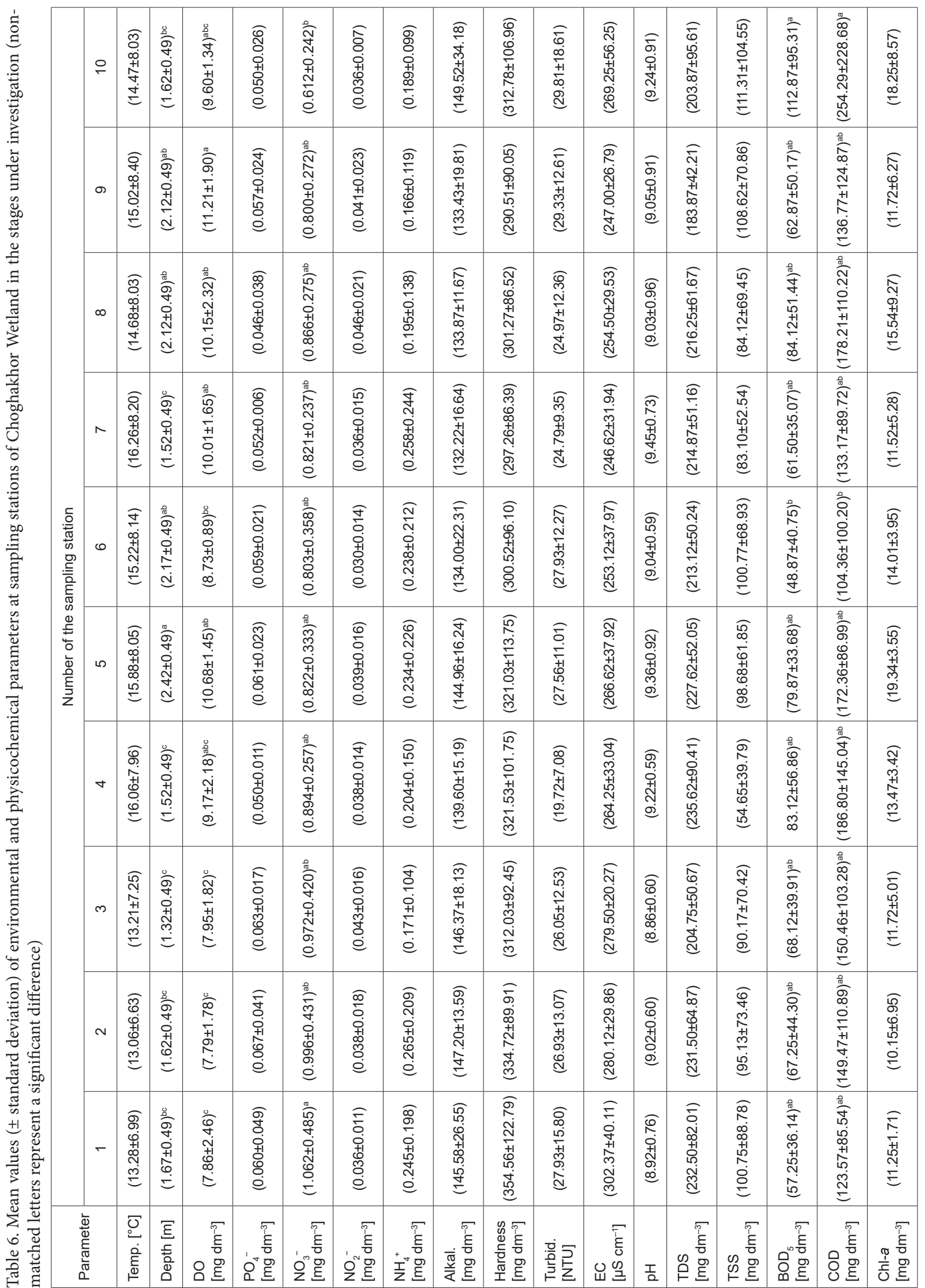




\begin{tabular}{|c|c|c|c|c|c|c|c|c|c|c|c|c|c|c|c|c|c|c|}
\hline & $\begin{array}{l}\frac{\alpha}{\alpha} \\
\Sigma \\
F\end{array}$ & 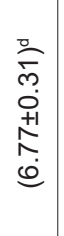 & 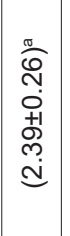 & $\begin{array}{l}0 \\
\stackrel{0}{\hat{D}} \\
0 \\
O \\
+1 \\
0 \\
\infty \\
\infty\end{array}$ & 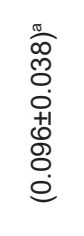 & 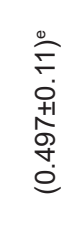 & 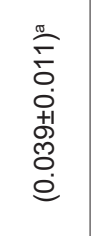 & $\begin{array}{l}0 \\
\frac{10}{10} \\
0 \\
0 \\
+1 \\
\dot{+1} \\
\dot{0} \\
\dot{0}\end{array}$ & 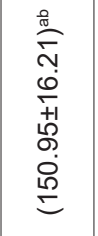 & 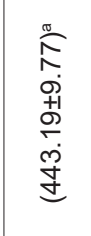 & $\begin{array}{l}\frac{\sigma}{\sigma} \\
0 \\
\infty \\
\infty \\
+1 \\
0 \\
0 \\
\infty \\
0 \\
0\end{array}$ & $\begin{array}{l}0 \\
\stackrel{0}{0} \\
\stackrel{0}{0} \\
0 \\
+1 \\
0 \\
0 \\
0 \\
\stackrel{0}{d}\end{array}$ & 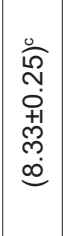 & 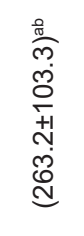 & 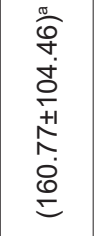 & 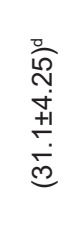 & 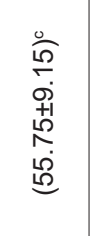 & 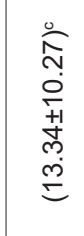 \\
\hline & $\begin{array}{l}z \\
\underset{乃}{\lessgtr} \\
\stackrel{2}{N}\end{array}$ & 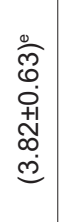 & 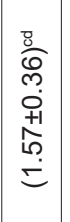 & $\begin{array}{l}\frac{0}{0} \\
\stackrel{0}{0} \\
0 \\
0+1 \\
+1 \\
0 \\
0 \\
0\end{array}$ & 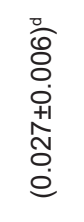 & 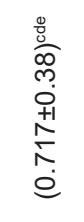 & 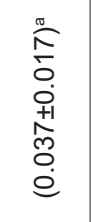 & 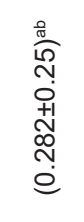 & 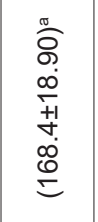 & 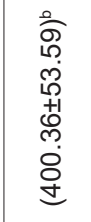 & 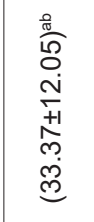 & 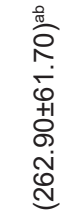 & 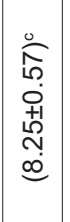 & 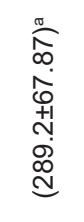 & 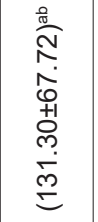 & 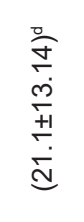 & 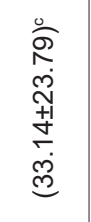 & 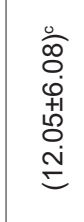 \\
\hline & $\begin{array}{l}\stackrel{\circlearrowright}{\breve{U}} \\
\check{F} \\
F\end{array}$ & 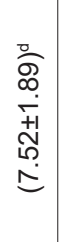 & 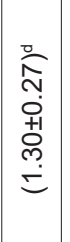 & 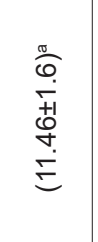 & 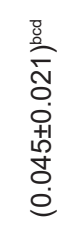 & 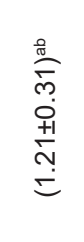 & 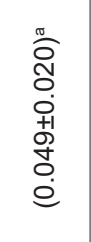 & $\begin{array}{l}\text { o } \\
\text { o. } \\
\dot{0} \\
+1 \\
0 \\
0 \\
0 \\
0\end{array}$ & $\begin{array}{l}0 \\
\stackrel{0}{0} \\
10 \\
0 \\
\stackrel{5}{+1} \\
0 \\
\stackrel{1}{0} \\
\stackrel{m}{=}\end{array}$ & 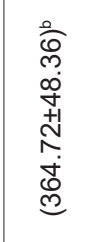 & 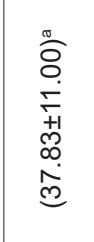 & 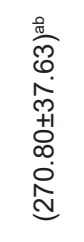 & 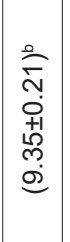 & 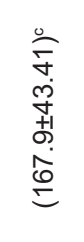 & 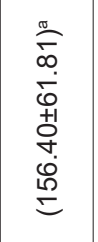 & 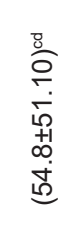 & $\begin{array}{l}0 \\
\stackrel{0}{0} \\
0 \\
o \\
0 \\
0 \\
+1 \\
\infty \\
\infty \\
0 \\
0 \\
0\end{array}$ & 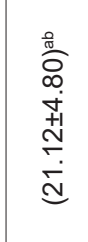 \\
\hline 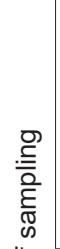 & 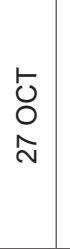 & 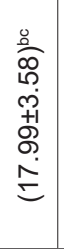 & 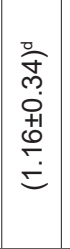 & 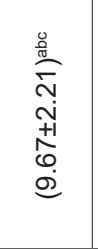 & 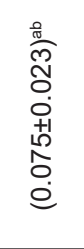 & $\begin{array}{l}\stackrel{\sigma}{10} \\
\text { m } \\
\stackrel{+}{+1} \\
\stackrel{+}{N} \\
=\end{array}$ & $\begin{array}{l}\frac{\pi}{0} \\
\vdots \\
0 \\
0 \\
+1 \\
\text { +1 } \\
0 \\
0 \\
0\end{array}$ & 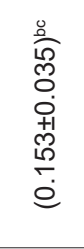 & 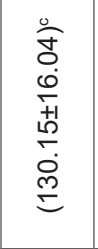 & 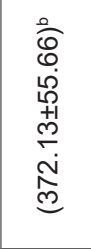 & 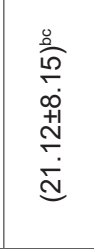 & 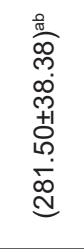 & 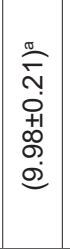 & 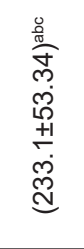 & 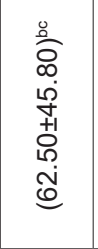 & 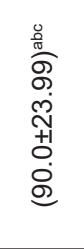 & 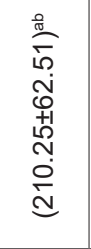 & 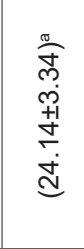 \\
\hline$\frac{\Phi}{\square}$ & $\begin{array}{l}\text { 岀 } \\
F \\
F\end{array}$ & 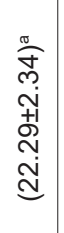 & 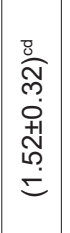 & \begin{tabular}{l}
$\stackrel{0}{0}$ \\
$\stackrel{0}{0}$ \\
\multirow{1}{*}{} \\
$\stackrel{0}{\circ}$ \\
$\infty$ \\
$\infty$
\end{tabular} & 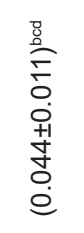 & $\begin{array}{l}\frac{0}{0} \\
\stackrel{5}{0} \\
\\
01 \\
0 \\
0 \\
0 \\
0\end{array}$ & 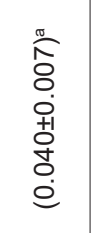 & 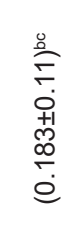 & 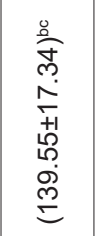 & 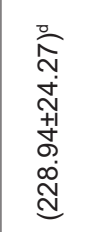 & 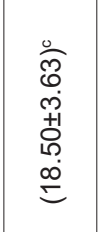 & 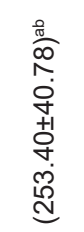 & 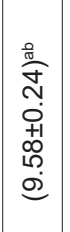 & 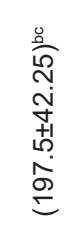 & 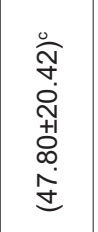 & 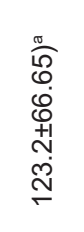 & 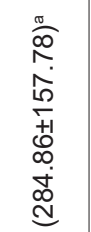 & $\begin{array}{l}\stackrel{0}{\stackrel{D}{N}} \\
\stackrel{\sim}{N} \\
\stackrel{+}{N} \\
\stackrel{\infty}{\infty} \\
\stackrel{\infty}{=}\end{array}$ \\
\hline & 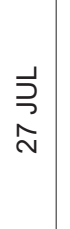 & 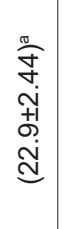 & 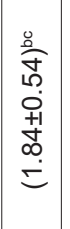 & 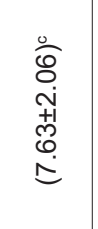 & 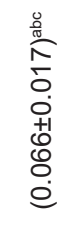 & 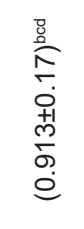 & 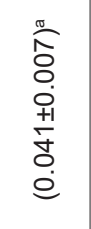 & $\begin{array}{l}0 \\
\stackrel{0}{0} \\
\dot{0} \\
\dot{+1} \\
\stackrel{+}{5} \\
\dot{0}\end{array}$ & $\begin{array}{l}\stackrel{0}{\widehat{N}} \\
\stackrel{+}{\dot{x}} \\
\stackrel{+}{+1} \\
\stackrel{N}{N} \\
\stackrel{N}{=}\end{array}$ & 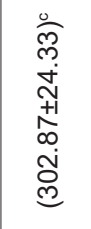 & 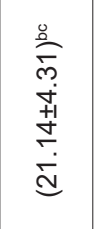 & 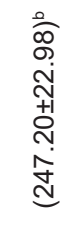 & $\mid \begin{array}{l}0 \\
\stackrel{0}{\sigma} \\
0 \\
0 \\
+1 \\
N \\
N \\
0 \\
0\end{array}$ & 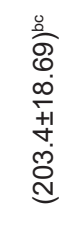 & 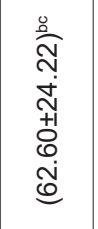 & 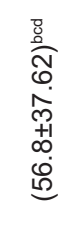 & 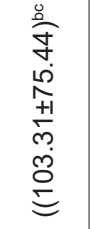 & 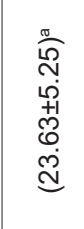 \\
\hline & $\begin{array}{l}\text { 그 } \\
\text { 음 }\end{array}$ & 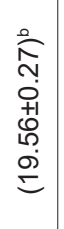 & 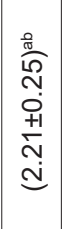 & $\begin{array}{l}0 \\
\stackrel{0}{0} \\
0 \\
+ \\
+1 \\
\stackrel{+1}{+} \\
\infty \\
\infty\end{array}$ & 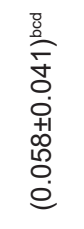 & $\begin{array}{l}\frac{8}{8} \\
\frac{0}{0} \\
\\
0 \\
+1 \\
\infty \\
\infty \\
0 \\
0\end{array}$ & $\begin{array}{l}\frac{\pi}{0} \\
0 \\
0 \\
0 \\
+1 \\
\hat{0} \\
0 \\
0 \\
0\end{array}$ & $\begin{array}{l}\stackrel{0}{\Im} \\
\stackrel{0}{0} \\
0 \\
0 \\
0 \\
0 \\
0\end{array}$ & $\begin{array}{l}\stackrel{0}{N} \\
\stackrel{0}{o} \\
+1 \\
\stackrel{N}{1} \\
\stackrel{N}{N} \\
\stackrel{N}{C}\end{array}$ & 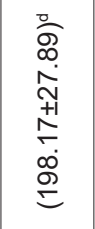 & 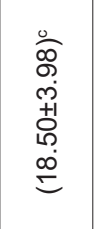 & 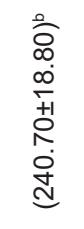 & 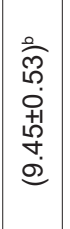 & 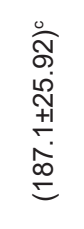 & 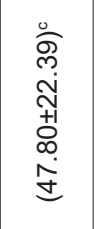 & 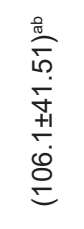 & 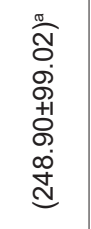 & 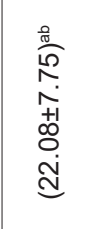 \\
\hline & $\begin{array}{l}\stackrel{\alpha}{a} \\
\stackrel{\alpha}{\alpha} \\
\stackrel{\infty}{N}\end{array}$ & 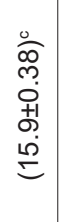 & 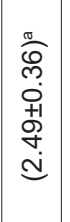 & 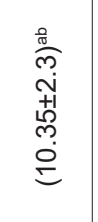 & 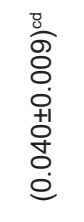 & 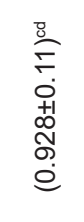 & $\begin{array}{l}0 \\
\bar{\theta} \\
\dot{0} \\
\dot{0} \\
+1 \\
\dot{+1} \\
\dot{0} \\
\dot{0}\end{array}$ & 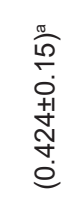 & $\begin{array}{l}0 \\
0 \\
0 \\
0 \\
0 \\
0 \\
0 \\
0 \\
0 \\
0 \\
0 \\
0 \\
=\end{array}$ & 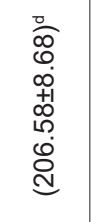 & 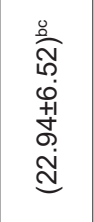 & 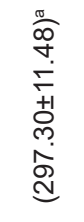 & 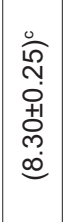 & 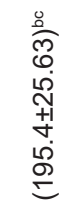 & 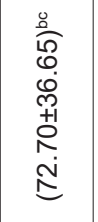 & 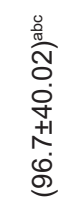 & 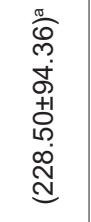 & 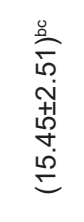 \\
\hline 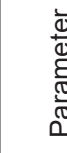 & & 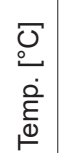 & 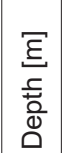 & 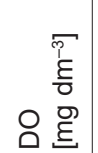 & 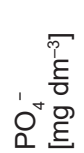 & 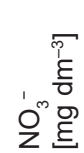 & 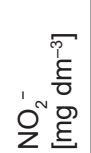 & 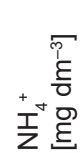 & 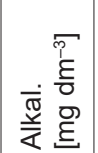 & 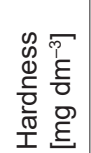 & 毫 & 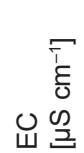 & I & 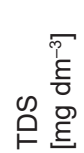 & 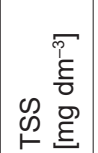 & 峞 & 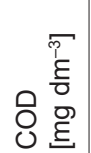 & 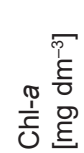 \\
\hline
\end{tabular}


that the high turbidity in the Koffe Wetland could be due to the fact that it has the highest wetland plant coverage. Ramesh and Jitendra (2009) stated that the lowest transparency was seen in summer. This was probably due to the high organic matter, algal bloom, silt and appearance of pulp. The Lowest turbidity was in spring and summer and the highest was in fall and winter. Suspended solid particles had an important role in increasing turbidity. Additionally, with the increasing amount of TSS during autumn and winter and the low growth of vegetation cover, which was effective in increasing suspended solids, turbidity was increased (USEPA 2000). Increasing water turbidity in autumn and winter was also due to strong winds during these seasons (especially fall) and shallow depth resulting from sediment mixture.

TSS changes were almost constant and uniform in spring and summer but they showed an ascending trend through autumn and winter. This could be due to the low growth of vegetation cover. Li et al. (2009) reported that the most effective factor in decreasing TSS in aqueous environments was high plant density, which could deposit the dissolved solids. This is one of the major advantages of wetlands as water transparency may be increased because these particles could be absorbed by plants (USEPA 2000).
The variation in the level of EC is attributed to the dissolved solids in water (Yimer and Mengistou 2009). So with the growing amounts of soluble solids (mostly salts), we were able to observe an increase in the EC. Therefore, TDS contents are directly related to the EC (Yimer and Mengistou 2009). According to the FAO recommendation, the acceptable TDS concentration for livestock drinking is between $100-1500 \mathrm{mg} \mathrm{dm}^{-3}$. Thus the range of TDS in this study was acceptable for livestock (167-289 $\mathrm{mg} \mathrm{dm}^{-3}$ ). TDS levels were generally stable in spring and summer. However, some minor changes were seen in autumn and winter. The TDS trend was generally similar to electrical conductivity. The highest amounts of BOD5 and COD were noted in spring and summer and the lowest in winter.

The required amount of $\mathrm{NO}_{3}^{-}$in water for livestock drinking use is set as $100 \mathrm{mg} \mathrm{dm}^{-3}$, however, for irrigation, the FAO recommends a maximum concentration of $30 \mathrm{mg} \mathrm{dm}^{-3}$, and a concentration below $5 \mathrm{mg}$ $\mathrm{dm}^{-3}$ poses no impact either on plants or soil (Yimer and Mengistou 2009). The $\mathrm{NO}_{3}{ }^{-}$concentration was between 0.497 and $1.24 \mathrm{mg} \mathrm{dm}^{-3}$. These values are much less than the minimum required set for livestock use. They are also less than the required concentration set for irrigation purposes. Therefore, the water in the wetland meets the standard requirements for livestock and

Table 8. List of benthic macroinvertebrates found at sampling stations in Choghakhor Wetland

\begin{tabular}{|c|c|c|c|c|c|c|c|c|c|c|c|c|}
\hline \multirow{2}{*}{ Class } & \multirow{2}{*}{ Order } & \multirow{2}{*}{ Family } & \multicolumn{10}{|c|}{ Stations } \\
\hline & & & 1 & 2 & 3 & 4 & 5 & 6 & 7 & 8 & 9 & 10 \\
\hline \multirow[t]{14}{*}{ Insecta } & Ephemeroptera & Baetidae & + & + & & + & + & & + & + & + & + \\
\hline & \multirow[t]{2}{*}{ Trichoptera } & Polycentropodidae & & & & & & + & & & & \\
\hline & & Rhyacophilidae & + & + & + & + & + & + & + & + & + & + \\
\hline & \multirow[t]{2}{*}{ Diptera } & Tabanidae & + & & & & & & & & & \\
\hline & & Chironomidae & + & + & + & + & + & + & + & + & + & + \\
\hline & \multirow[t]{5}{*}{ Coleoptera } & Curculionidae & + & & & & & + & & & & \\
\hline & & Hydrophilidae & & & & & & & + & & & \\
\hline & & Elmidae & & & & & & & & + & & \\
\hline & & Haliplidae & & + & & & & + & & & & + \\
\hline & & Dytiscidae & & & + & & & & & & & \\
\hline & \multirow[t]{4}{*}{ Odonata } & Coenagriidae & + & + & + & + & + & + & + & + & + & + \\
\hline & & Agridae & & & & & + & & + & + & & \\
\hline & & Cordulegasteridae & + & & & + & & + & & + & + & + \\
\hline & & Gomphidae & & & & & & + & & & & \\
\hline Crustacea & Amphipoda & Gammaridae & + & & & + & + & + & + & + & + & + \\
\hline Bivalvia & Lamellibranchiata & Sphaeridae & + & + & + & + & + & + & + & + & + & + \\
\hline \multirow[t]{5}{*}{ Gastropoda } & \multirow[t]{2}{*}{ Pulmonata } & Limnaeidae & + & + & + & + & + & + & + & + & + & + \\
\hline & & Planorbidae & & + & + & & + & + & & & & \\
\hline & \multirow[t]{3}{*}{ Porosobranchiata } & Viviparidae & + & + & + & + & + & + & + & + & + & + \\
\hline & & Hydrobiidae & & + & + & & & & & + & & + \\
\hline & & Valvatidae & + & + & + & + & + & + & + & + & + & + \\
\hline \multirow[t]{4}{*}{ Oligochaeta } & \multirow[t]{2}{*}{ Tubificida } & Tubificidae & + & + & + & + & + & + & + & + & + & + \\
\hline & & Naididae & + & + & + & + & + & + & + & + & + & \\
\hline & Lumbricida & Lumbricidae & + & & + & & & & & & & \\
\hline & Haplotaxida & Haplotaxidae & + & + & + & & + & + & + & & + & + \\
\hline
\end{tabular}


agricultural uses from the $\mathrm{NO}^{-3}$ concentration point of view. This might suggest that there are active $\mathrm{N}$ - fixing bacterias or wetland plants that utilize nitrates, thereby reducing the $\mathrm{NO}^{-3}$ concentration. Our study results are consistent with the results of Yimer and Mengistou 2009.

As studies indicate, $\mathrm{PO}_{4}^{-3}$ tends to be fixed to soil particles and therefore reaches water bodies through soil erosion. Phosphates enter water bodies through man-made routes and contribute to surface water pollution due to algal blooms (Yimer and Mengistou 2009). According to Ayers and Westcot (1985), the maximum allowable concentration of phosphate for irrigation water is $2 \mathrm{mg} \mathrm{dm}^{-3}$. The mean concentration values $\left(0.027-0.096 \mathrm{mg} \mathrm{dm}^{-3}\right)$ of phosphate measured are all well below the recommended level for the use of water for irrigation purposes.

\section{Benthic macroinvertebrates}

In total, 25 families of Benthic macroinvertebrate (5 classes and 12 orders) were identified in Choghakhor Wetland (Tables 8 and 9).

The outcome dendrogram of cluster analysis (braycurtis distance and single linkage) at different stations based on the presence and absence of 25 identified families (Fig. 3) showed that all stations except 6 were

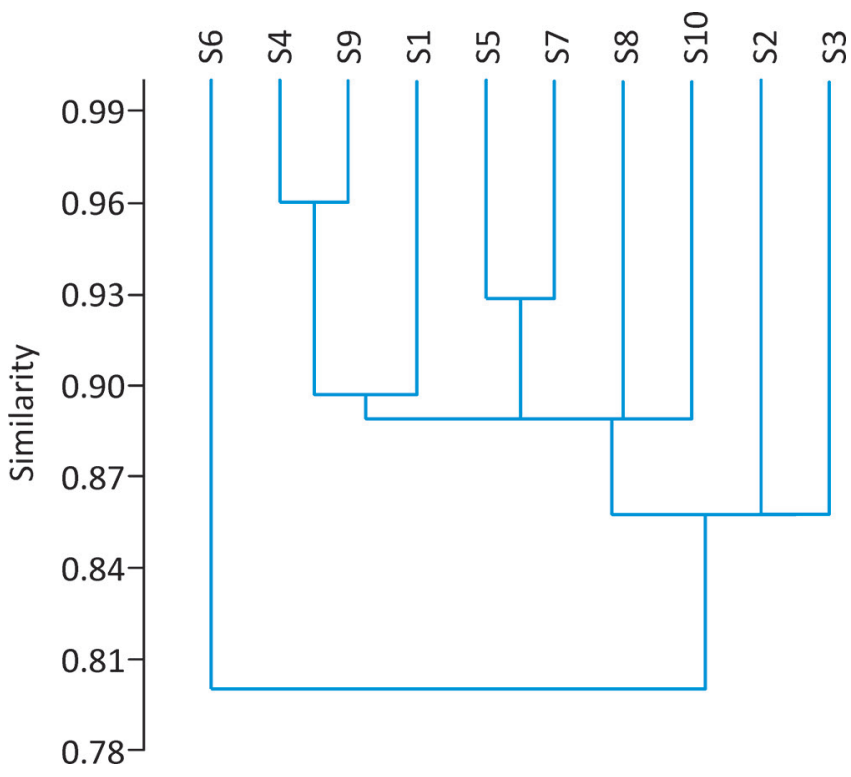

Fig. 3. the analysis of the Bray-Curtis faunistic similarity between macrobenthic assemblages inhabiting different sampling stations

in one cluster. Stations 4 and 9 with $96 \%$ similarity and stations 2 and 3 with $86 \%$ had the most and least similarity in this cluster, respectively. Also, station 6 is connected to this cluster separately and has the least similarity to other stations. As can be seen, all stations

Table 9. List of benthic macroinvertebrates found in sampling stages in Choghakhor Wetland

\begin{tabular}{|c|c|c|c|c|c|c|c|c|c|c|}
\hline \multirow{2}{*}{ Class } & \multirow{2}{*}{ Order } & \multirow{2}{*}{ Family } & \multicolumn{8}{|c|}{ Stages } \\
\hline & & & 25 APR & 10 JUN & 27 JUL & 11 SEP & 27 OCT & 11 DEC & 25 JAN & 11 MAR \\
\hline \multirow[t]{14}{*}{ Insecta } & Ephemeroptera & Baetidae & + & & + & + & + & + & + & + \\
\hline & \multirow[t]{2}{*}{ Tricoptera } & Polycentropodidae & & & & & + & & & \\
\hline & & Rhyacophilidae & + & & + & + & + & + & + & + \\
\hline & \multirow[t]{2}{*}{ Diptera } & Tabanidae & + & & & & & & & \\
\hline & & Chironomidae & + & + & + & + & + & + & + & + \\
\hline & \multirow[t]{5}{*}{ Coleoptera } & Curculionidae & + & & & + & & & & \\
\hline & & Hydrophilidae & + & & & & & & & \\
\hline & & Elmidae & & & + & & & & & \\
\hline & & Haliplidae & & + & + & + & & & & \\
\hline & & Dytiscidae & + & & & & & & & \\
\hline & \multirow[t]{4}{*}{ Odonata } & Coenagriidae & + & + & + & + & + & + & + & + \\
\hline & & Agridae & + & + & + & & & & + & \\
\hline & & Cordulegasteridae & + & + & & & + & + & + & \\
\hline & & Gomphidae & & & + & & & & & \\
\hline Crustacea & Amphipoda & Gammaridae & + & + & + & + & + & & + & \\
\hline Bivalvia & Lamellibranchiata & Sphaeridae & + & + & + & + & + & + & + & + \\
\hline \multirow[t]{5}{*}{ Gastropoda } & \multirow[t]{2}{*}{ Pulmonata } & Limnaeidae & + & + & + & + & + & + & + & + \\
\hline & & Planorbidae & & & & + & + & + & + & + \\
\hline & \multirow[t]{3}{*}{ Porosobranchiata } & Viviparidae & + & + & + & + & + & + & + & + \\
\hline & & Hydrobiidae & & + & + & & & & + & \\
\hline & & Valvatidae & + & + & + & + & + & + & + & + \\
\hline \multirow[t]{4}{*}{ Oligochaeta } & \multirow[t]{2}{*}{ Tubificida } & Tubificidae & + & + & + & + & + & + & + & + \\
\hline & & Naididae & + & + & + & + & & + & + & \\
\hline & Lumbricida & Lumbricidae & + & & & & & & + & \\
\hline & Haplotoxida & Haplotaxidae & + & + & + & & + & & & + \\
\hline
\end{tabular}




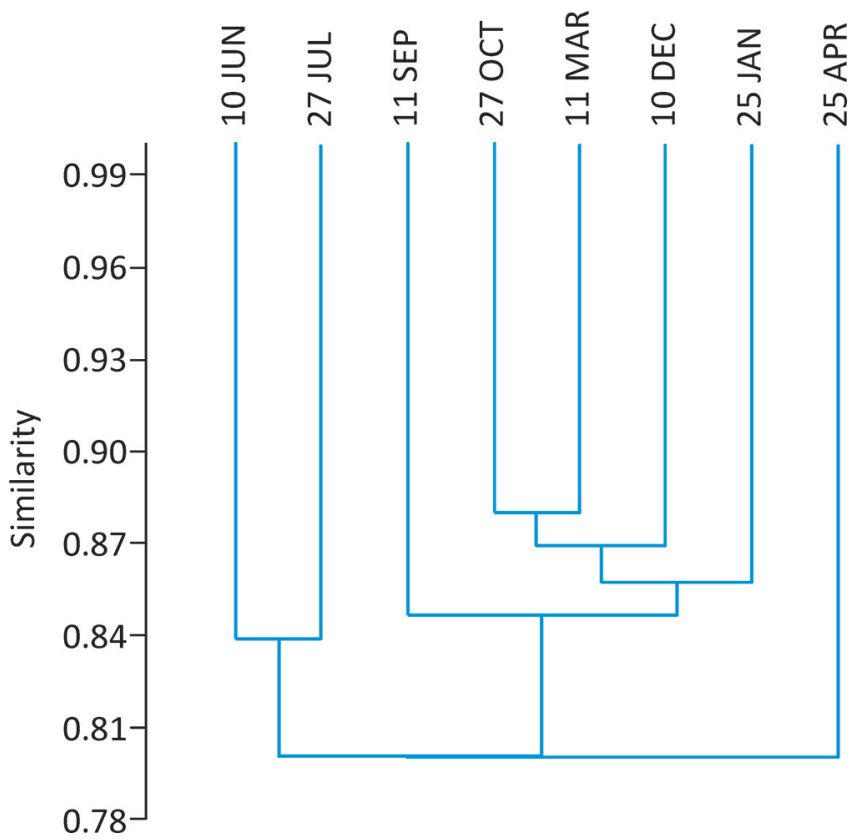

Fig 4. the analysis of the Bray-Curtis faunistic similarity between macrobenthic assemblages inhabiting different sampling stages have high similarity and there is not much difference in the terms of organism communities. The studied area's uniformity and similar characteristics of habitat and physico chemical parameters could be the reason for this result.

The outcome dendrogram of the cluster analysis (bray-curtis distance and single linkage) at different stages based on the presence and absence of 25 identified families (Fig. 4) showed that the samples from June and July, stages 2 and 3 with $84 \%$ similarity are in one cluster and the samples from September, October, December, January and March 4, 5, 6, 7 and 8 are in another cluster, respectively. The samples from October and March with $88 \%$ similarity are in the second cluster stages 5 and 8 with $88 \%$ similarity and those of September stage 4 had the most and least similarity, respectively. The samples from April Stage 1 are attached to these two clusters separately and have the least similarity to the others.

The density of Choghakhr Wetland's benthic macroinvertebrates was also determined for each family in the sampling stages (Table 10). The maximum and minimum abundance of population were gastropoda

Table 10. The mean density and percentage of abundance of Choghakhor Wetland's benthic macroinvertebrates at sampling stages (total of 10 stations)

\begin{tabular}{|c|c|c|c|c|c|c|c|c|c|c|c|c|}
\hline \multirow{3}{*}{ Family } & \multicolumn{3}{|c|}{ Spring } & \multicolumn{3}{|c|}{ Summer } & \multicolumn{3}{|c|}{ Autumn } & \multicolumn{3}{|c|}{ Winter } \\
\hline & \multicolumn{2}{|c|}{ Density [ind. $\mathrm{m}^{-2}$ ] } & \multirow{2}{*}{ Percent } & \multicolumn{2}{|c|}{ Density [ind. $\mathrm{m}^{-2}$ ] } & \multirow{2}{*}{ Percent } & \multicolumn{2}{|c|}{ Density [ind. $\mathrm{m}^{-2}$ ] } & \multirow{2}{*}{ Percent } & \multicolumn{2}{|c|}{ Density [ind. $\mathrm{m}^{-2}$ ] } & \multirow{2}{*}{ Percent } \\
\hline & $25 \mathrm{APR}$ & 10 JUN & & $27 \mathrm{JUL}$ & 11 SEP & & 27 OCT & $11 \mathrm{DEC}$ & & 25 JAN & 11 MAR & \\
\hline Baetidae & 27 & 3 & 0.29 & 12 & 30 & 0.54 & 54 & 18 & 0.48 & 24 & 12 & 0.41 \\
\hline Polycentropodidae & 0 & 0 & 0 & 0 & 0 & 0 & 9 & 0 & 0.06 & 0 & 0 & 0 \\
\hline Rhyacophilidae & 39 & 0 & 0.37 & 57 & 29 & 1.23 & 117 & 141 & 1.73 & 39 & 66 & 1.20 \\
\hline Tabanidae & 6 & 0 & 0.05 & 0 & 0 & 0 & 0 & 0 & 0 & 0 & 0 & 0 \\
\hline Chironomidae & 453 & 306 & 7.35 & 174 & 96 & 3.48 & 84 & 267 & 2.36 & 1218 & 1401 & 29.96 \\
\hline Curculionidae & 3 & 0 & 0.02 & 0 & 3 & 0.03 & 0 & 0 & 0 & 0 & 0 & 0 \\
\hline Hydrophilidae & 3 & 0 & 0.02 & 0 & 3 & 0.03 & 0 & 0 & 0 & 0 & 0 & 0 \\
\hline Elmidae & 0 & 0 & 0 & 27 & 0 & 0.34 & 0 & 0 & 0 & 0 & 0 & 0 \\
\hline Haliplidae & 0 & 12 & 0.05 & 12 & 6 & 0.23 & 0 & 0 & 0 & 0 & 0 & 0 \\
\hline Dytiscidae & 6 & 0 & 0.05 & 0 & 0 & 0 & 0 & 0 & 0 & 0 & 0 & 0 \\
\hline Coenagriidae & 324 & 321 & 6.25 & 225 & 225 & 5.80 & 210 & 243 & 3.04 & 198 & 57 & 2.91 \\
\hline Agridae & 15 & 12 & 0.29 & 6 & 0 & 0.07 & 0 & 0 & 0 & 6 & 0 & 0.06 \\
\hline Cordulegasteridae & 24 & 30 & 0.52 & 0 & 6 & 0.07 & 15 & 6 & 0.14 & 9 & 0 & 0.10 \\
\hline Gomphidae & 0 & 0 & 0 & 6 & 0 & 0.07 & 0 & 0 & 0 & 0 & 0 & 0 \\
\hline Gammaridae & 105 & 87 & 1.86 & 60 & 63 & 1.58 & 30 & 0 & 0.20 & 12 & 0 & 0.13 \\
\hline Sphaeridae & 363 & 276 & 6.19 & 288 & 388 & 8.74 & 972 & 411 & 9.30 & 51 & 243 & 3.36 \\
\hline Limnaeidae & 468 & 390 & 8.31 & 378 & 300 & 8.74 & 1152 & 534 & 11.34 & 351 & 330 & 7.79 \\
\hline Planorbidae & 0 & 0 & 0 & 0 & 21 & 0.27 & 12 & 6 & 0.12 & 27 & 66 & 1.06 \\
\hline Viviparidae & 168 & 105 & 2.64 & 81 & 30 & 1.43 & 465 & 318 & 5.26 & 114 & 207 & 3.67 \\
\hline Hydrobiidae & 0 & 24 & 0.23 & 30 & 0 & 0.38 & 0 & 0 & 0 & 6 & 0 & 0.06 \\
\hline Valvatidae & 2692 & 2016 & 45.69 & 2073 & 2594 & 59.92 & 6870 & 2646 & 64.02 & 1134 & 1107 & 25.64 \\
\hline Tubificidae & 582 & 687 & 12.30 & 321 & 156 & 6.15 & 111 & 147 & 1.73 & 450 & 1146 & 18.26 \\
\hline Naididae & 273 & 18 & 2.82 & 21 & 27 & 0.61 & 0 & 18 & 0.12 & 174 & 0 & 1.99 \\
\hline Lumbricidae & 45 & 0 & 0.43 & 0 & 0 & 0 & 0 & 0 & 0 & 27 & 0 & 0.30 \\
\hline Haplotaxidae & 315 & 114 & 4.15 & 15 & 0 & 0.19 & 6 & 0 & 0.04 & 0 & 264 & 3.02 \\
\hline Total & 5918 & 4390 & 100 & 3786 & 3969 & 100 & 10107 & 4755 & 100 & 3840 & 4899 & 100 \\
\hline
\end{tabular}


with $61.66 \%$ and crustaceans with $0.94 \%$ respectively. The maximum density of macroinvertebrates 14862 ind. $\mathrm{m}^{-2}$ was observed in autumn (October and December stages 5 and 6 ) and the minimum 7755 ind. $\mathrm{m}^{-2}$ in summer (July and September stages 3 and 4 ). Seasonal changes in environmental conditions, life cycle of some benthic organisms and consumption by fish (especially in spring and summer) and aquatic birds (especially in winter and spring) are the reasons for the decrease in the density and biomass of macroinvertebrates.

Flow velocity and sediment type were related to the kind of environment, influencing taxa distribution, their abundance, richness and diversity (Alvarez-Mieles et al. 2013). No changes in measured indices were observed at any stations $(\mathrm{p}<0.05)$. No observed significant differences for these parameters between different sites could be related to the same environmental conditions. For example, Ramesh and Jitendra (2009) used the Shannon diversity to evaluate Asan wetland. The range of this index for different stations throughout the year was between $61 / 4-50 / 3$, which was completely uniform. Also, Nemati et al (2009) examined the annual average of the Shannon index in the Zayandehrud river and reported it to be between 0.8 and 1.5. Yimer and Mengistou 2009 found that the vegetated Koffe wetland seems to have the lowest Shannon-Weiner evenness for macroinvertebrates. This might show that vegetation cannot be a factor in determining the highest macroinvertebrate diversity. However, distributions of plant species can affect invertebrate distributions. For example, different invertebrate communities are associated with different types of wetland plants. According to Heino (2000), microenvironment species are lowest in wetlands with few aquatic plants. This might be the amount of organic substrata that could influence the diversity of macroinvertebrates. The presence of submerged and emergent macrophytes such as Typhaceae provided an increase in the surface area for biofilm developments, food for macroinvertebrates, and a habitat for predators and other invertebrates requiring vegetation for oviposition. Richness index changes at different stages of sampling were significant $(\mathrm{p}<0.01)$. The highest average of households was noted in the first stage of sampling (April) and the lowest number of families was seen in the late autumn (December) and the late winter (March) (Fig. 5). There was a downward trend in the richness index through spring and winter, which was due to some changes in physical and chemical parameters in the composition of the substrate, the life cycle of insects and other creatures and the reduction of nutrients. When the water quality and habitat conditions got better, the richness index rose (Sharma et al. 2006). Although total richness was one of the indicators of environmental pressures, when pollution increased, this index fell, but when the entrance of organic materials was high, the total richness also increased (Sharma et al. 2006). Moreover, habitat diversity and growth of plants could have resulted in an increasing index at some stations.

Margalef diversity changes were observed at different stages of sampling (Fig. 6). The general trend from April (early spring) to March (late winter) was downward and there was a statistically significant difference between them $(\mathrm{p}<0.01)$.

Simpson diversity changes at different stages of sampling are in Figure 7. As can be seen, from April

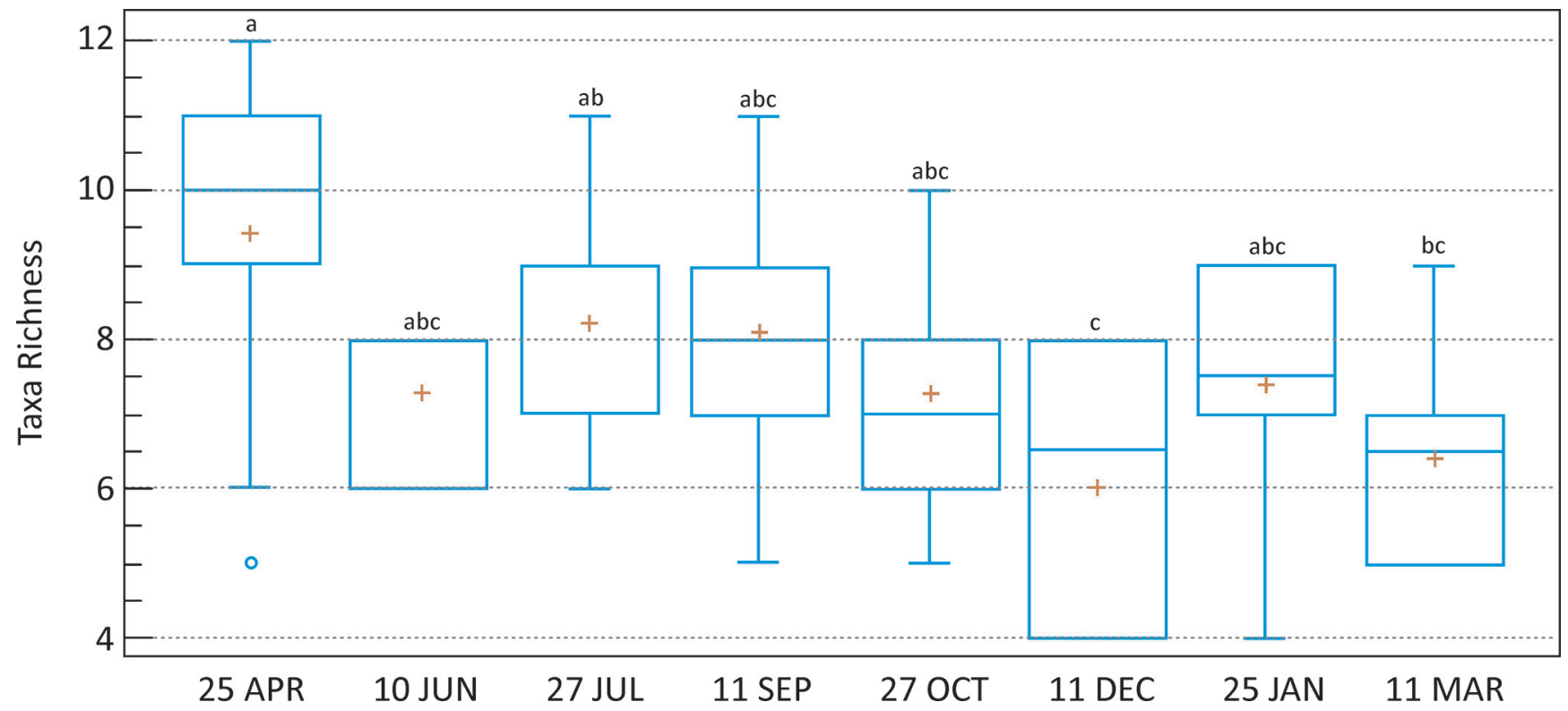

Fig. 5. Changes of taxa richness in various stages of sampling (non-matched letters represent a significant difference) 
(early spring) to March (late winter) there was a slight downward trend $(\mathrm{p}<0.05)$ with a tangible reduction in September and October. In general, all three diversity indices (Shannon, Margalef and Simpson) and richness indices decreased from spring to winter. They were compatible with each other. The Shannon diversity index was used to evaluate diversity in communities. It showed that the diversity of identified families in the wetlands decreased in spring towards winter, whereas the Simpson diversity index provided information about the composition and structure of communities (Li et al. 2009).
Shannon diversity changes at different stages of sampling are shown in Figure 8. The Shannon diversity showed a significant difference at all stages $(p<0.01)$. As can be seen, the trend of the first stage of sampling (spring) until the $8^{\text {th }}$ stage (winter) was descending. A large number of families of invertebrates were compatible with this index. According to Gencer and Nilgun (2010) Values above 3.0 indicate that habitat structure is stable and balanced and values under 1.0 indicate the presence of pollution and degradation of habitat structure. On the basis of these criteria, the Shannon diversity index for all stages was below 1.9, further indicating the presence of

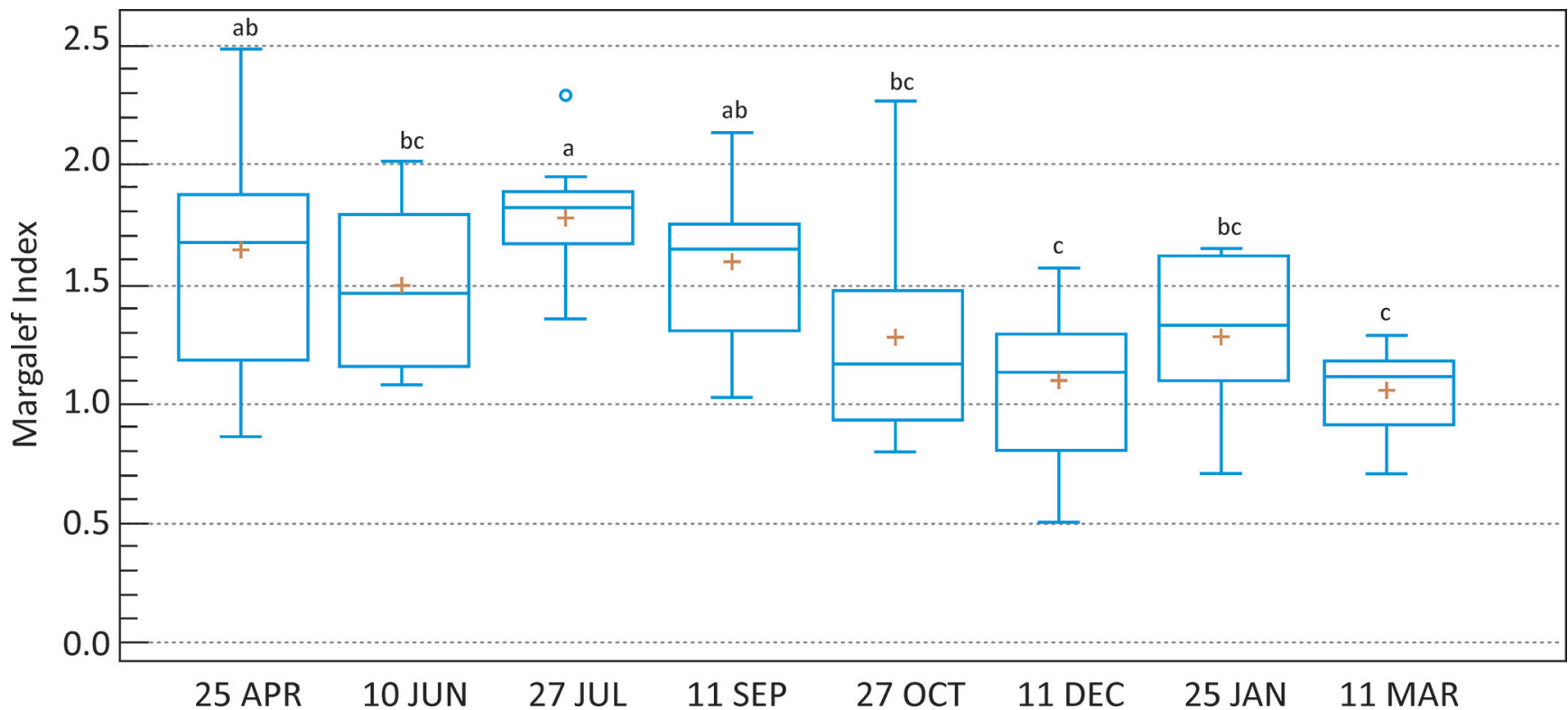

Fig. 6. Changes of Margalef's index in various stages of sampling (non-matched letters represent a significant difference)

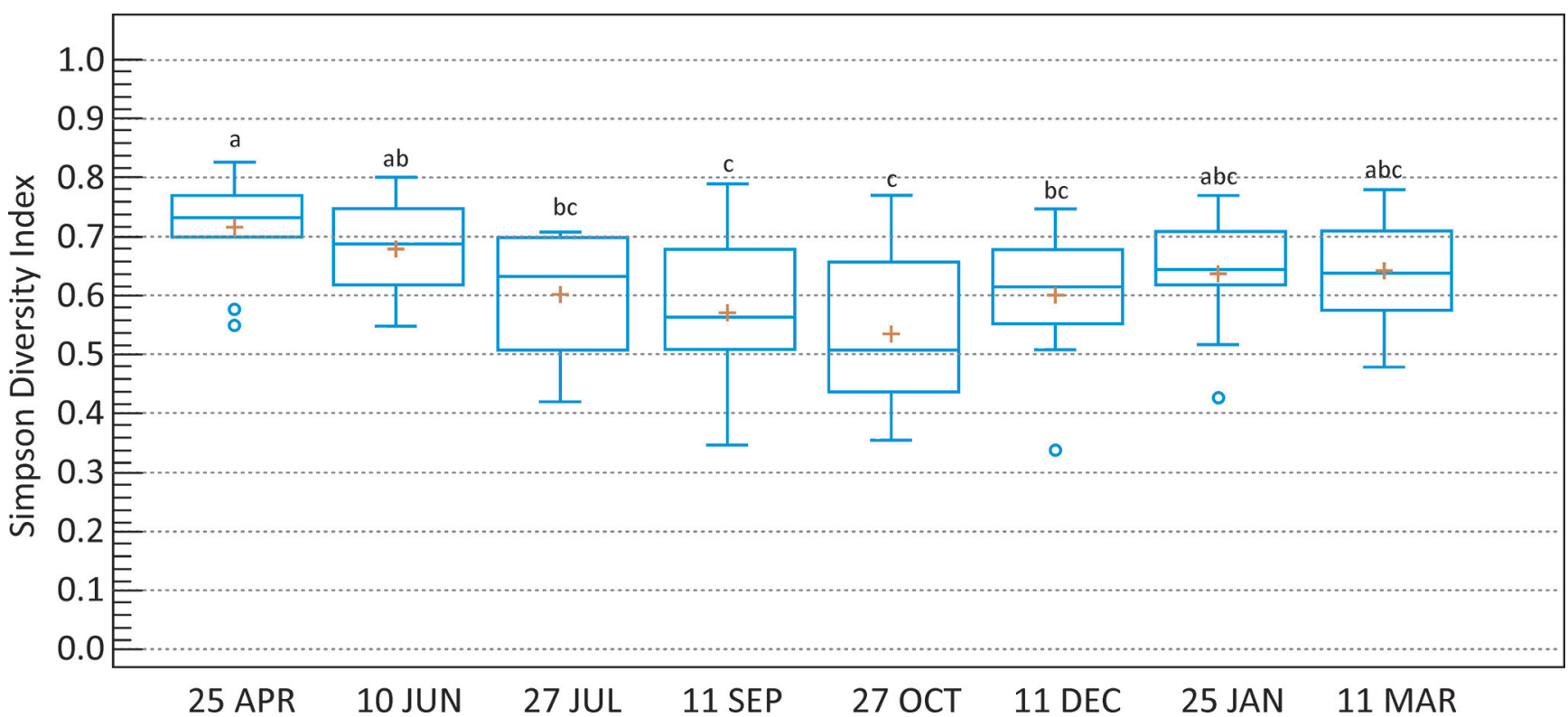

Fig. 7. Changes of Simpson diversity index in various stages of sampling (non-matched letters represent a significant difference) 
pollution and degradation of habitat structure. The reduction of Shannon diversity could have been caused by factors such as changes in physicochemical factors, substrate composition changes, better food conditions and so on. The low growth of plants from spring to winter and the loss of an efficient habitat for these species could be also a reason for the reduction in variability. One of the reasons for the decline in the diversity of organisms in the river was considered to be the reduction of plant growth (Nemati et al. 2009). It is possible to use a variety of indicators for water quality assessments based on the assumption that the structure of the Benthic commu- nities would change along with environmental disturbances. The Shannon-Wiener diversity index had a low accuracy due to some misjudgments and the limitation of the sampling area (the small area of the grab) (Taowu et al. 2008). In addition, this index was more influenced by nutrients and better oxygen conditions (Nemati et al. 2009; Ramesh and Jitendra 2009).

As for other indicators, the Hilsenhoff biological index in various stages of sampling was statistically significant $(\mathrm{p}<0.05)$. The highest and lowest rate of Hilsenhoff was calculated at the second and sixth stages of sampling (Fig. 9). Water had a poor quality at the

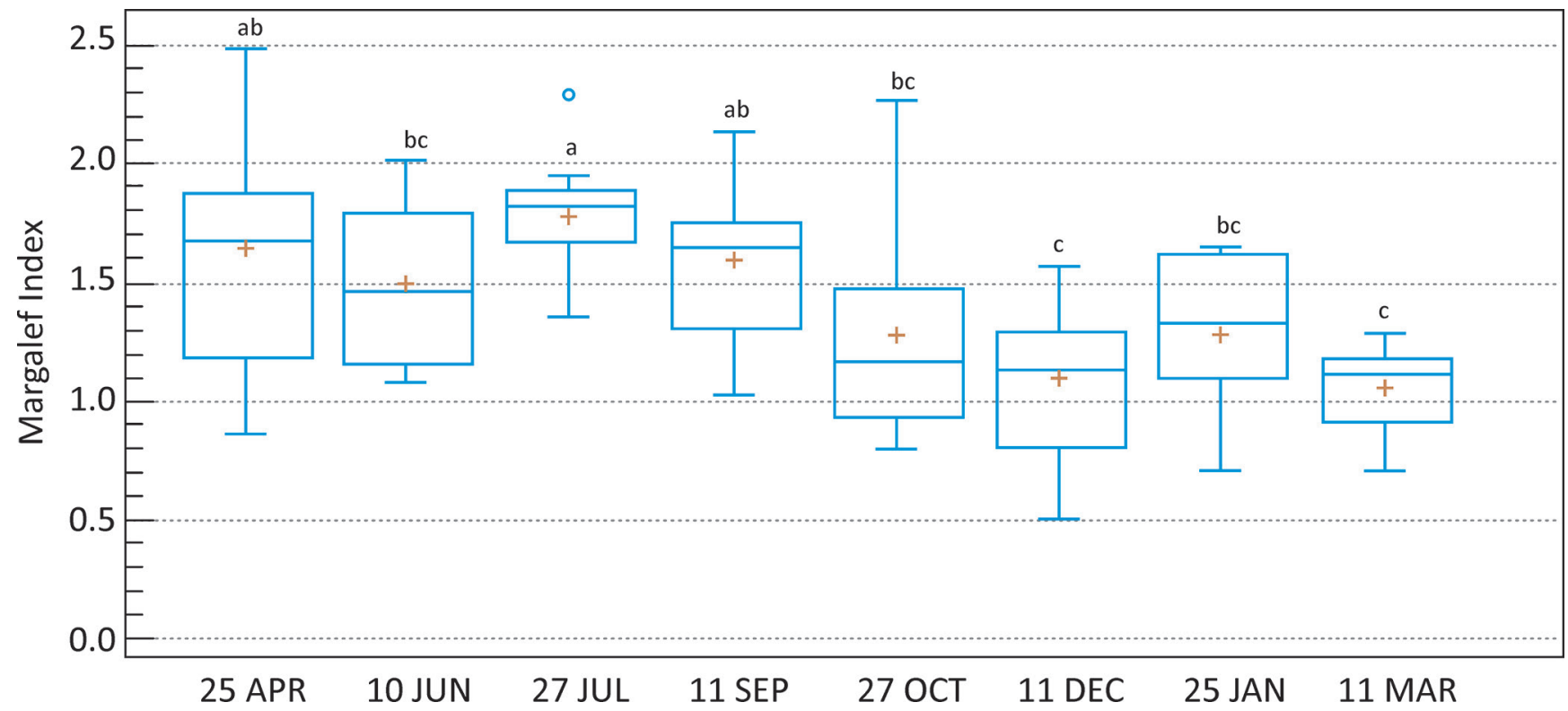

Fig. 8. Changes of Shannon-Wiener diversity index in various stages of sampling (non-matched letters represent a significant difference)

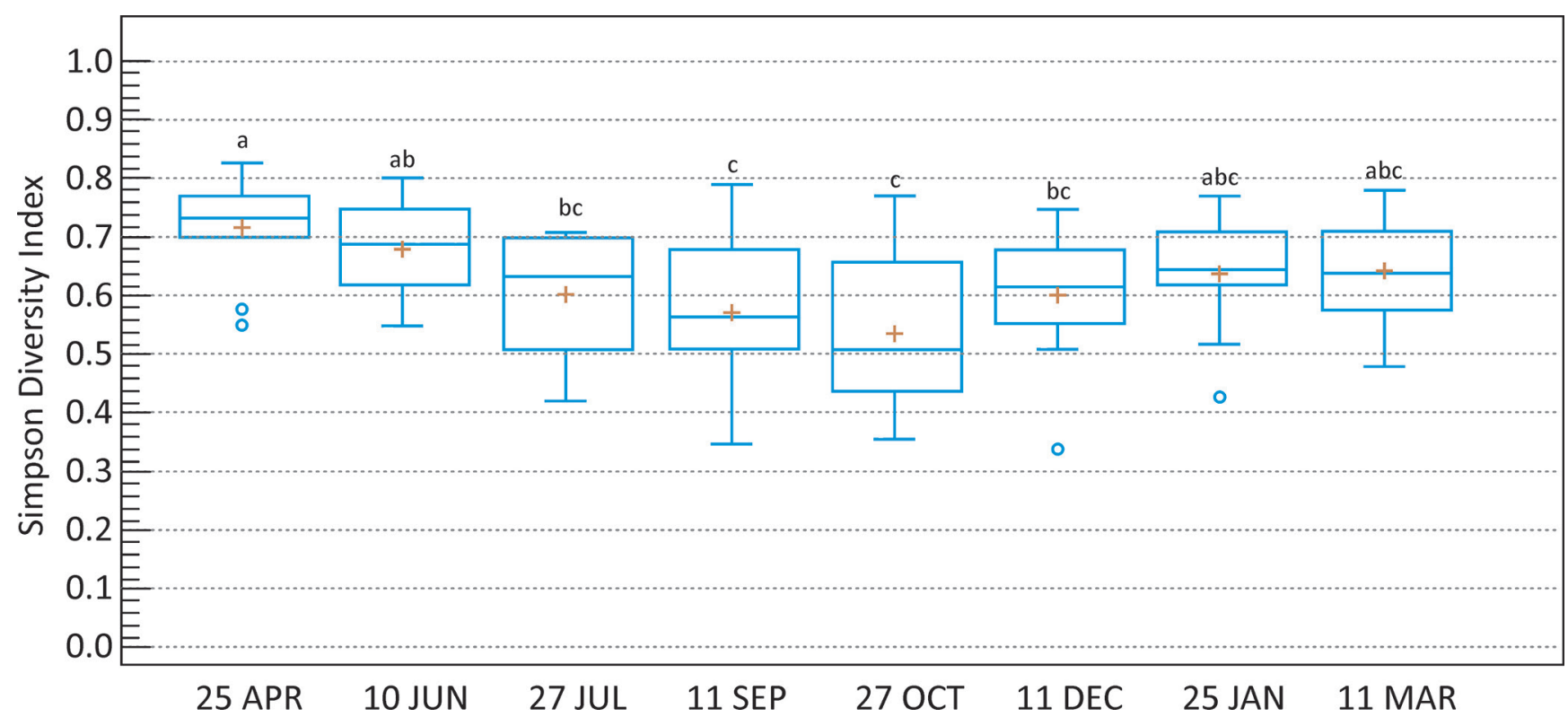

Fig. 9. Changes of HBI in various stages of sampling (non-matched letters represent a significant difference) 
second stage, and in other stages it had a relatively poor quality (most likely an indication of substantial organic pollution). This could be due to the greater diversity of gastropoda shells at this stage compared to other stages. In aquatic ecosystems, soil contamination can be created at a particular time and place; however, these changes are limited. But they could be clearly visible with the help of the population structure. This might result in changes in species diversity and an increase or decrease in the value of different indices. HBI is a more reliable indicator than diversity indices; moreover, this index is associated with a higher tolerance level of ac- curacy and it does not require complicated calculations (Taowu et al. 2008). Tolerance of a taxon is based on its ability to survive in the short and long term under the influence of physicochemical stress, which could be due to chemical pollution, habitat destruction and hydrological changes (Taowu et al. 2008).

BMWP changes were also significant $(\mathrm{p}<0.01)$ at different stages of sampling and times (Fig. 10). The highest and lowest values of this index were evaluated at stages 1 (early spring) and 8 (late winter), respectively. There was a downward trend from spring to winter, but $50 \%$ of the highest data BMWP in Stage 2

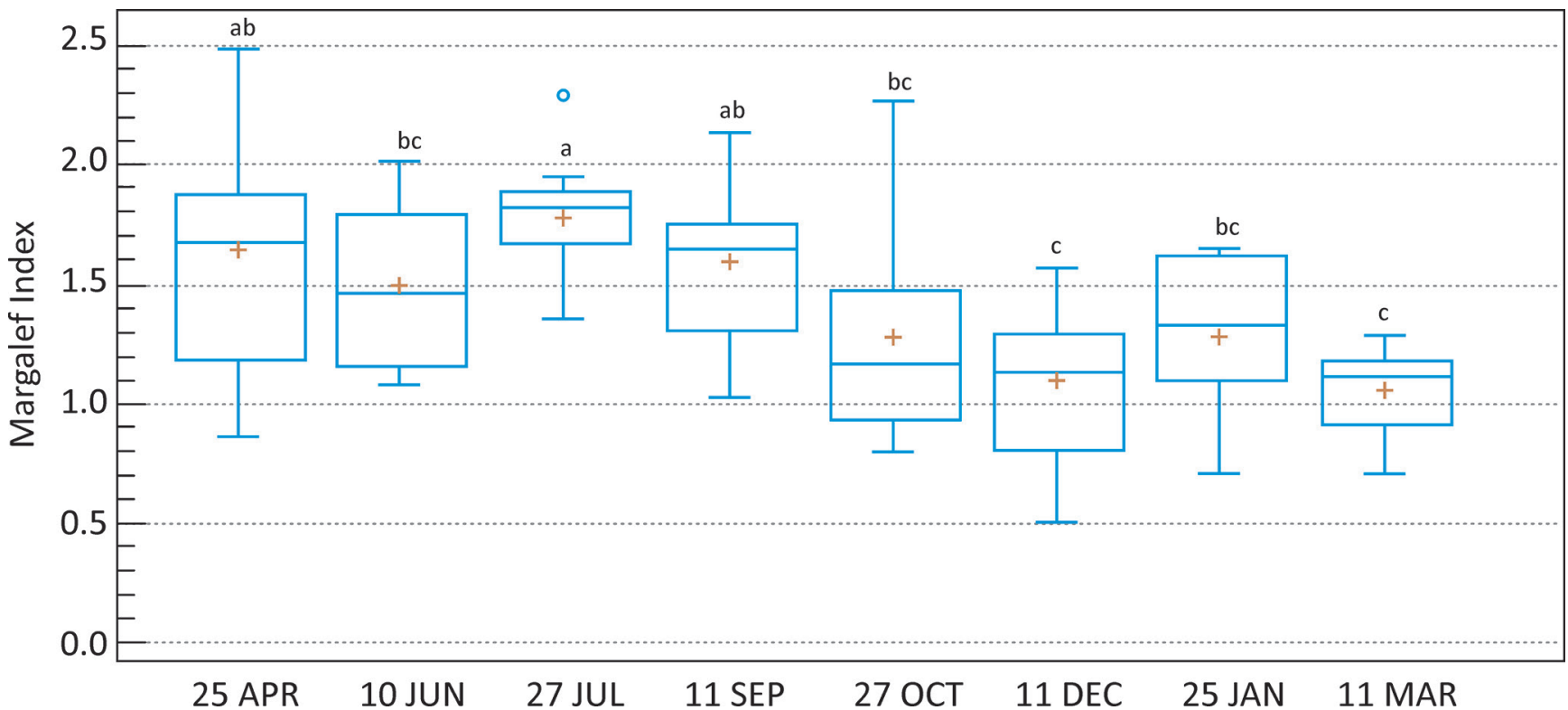

Fig. 10. Changes of BMWP in various stages of sampling (non-matched letters represent a significant difference)

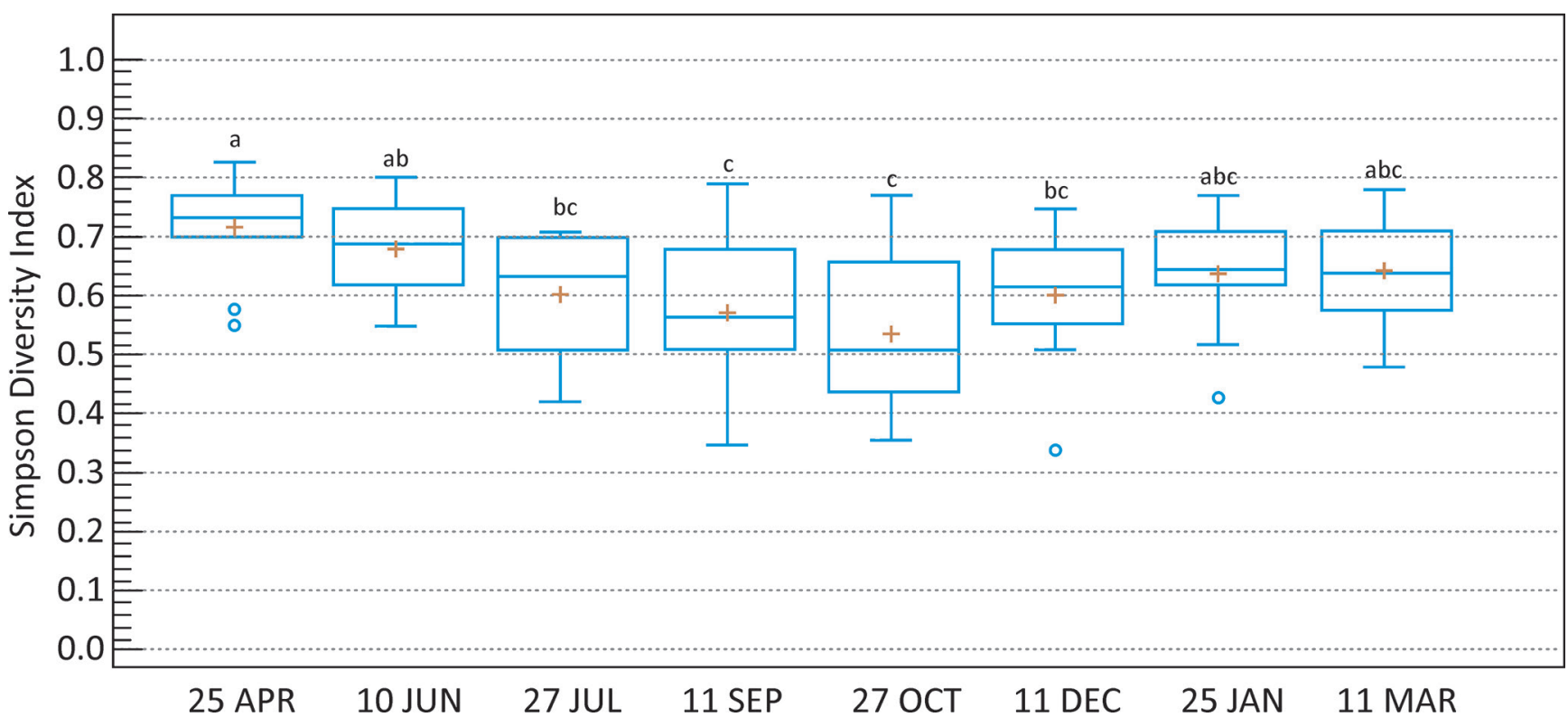

Fig. 11. Changes of ASPT in various stages of sampling (non-matched letters represent a significant difference) 
(late spring) was lower than in other stages (Fig. 10). The absence of any clear difference between the various stages of sampling was one of the weaknesses of the biological indices at family level (Nemati et al. 2009). Although the changes could be due to habitat changes over time or even changes in water quality, they may not be enough to replace the other families (Nemati et al. 2009). Moreover, several orders of ephemeroptera and plecoptera did not exist. Furthermore, the absence of plecoptera sensitive to pollutants (Table 11) showed that the BMWP index assessment was correct. Among different stages of sampling, the water quality of stage 1 was of average class and for the other stages, it was assigned to the bad quality class. BMWP and ASPT indices have been used to evaluate other wetlands and rivers (Bode et al. 1996; Czeniawska-Kusza 2005; Batty et al. 2005; Sharma et al. 2006; Galbrand et al. 2007; Nemati et al. 2009). The results showed that BMWP and ASPT indices were suitable for the evaluation of water quality.

There were significant differences $(\mathrm{p}<0.05)$ in ASPT between various stages of sampling (Fig. 11). We could see that the highest and lowest amounts of ASPT were at stage 6 (early fall), stage 8 (late winter) and stage 2 (late spring). This is based on the fact that water quality was in 2 categories of severity (stage 2 and 8 ) and average stages (other stages). All the chosen stations were the same in terms of physical structure; however, the differences could have resulted from micro-habitat differences in each station, plant growth and also, the diversity of benthic families and finally, the probability of benthic settlements. Hsu et al. (2011) revealed that increasing vegetation could be the reason for the increase in the richness and density of benthic macroinvertebrates. They stated that the most important factors controlling the variation in the protected wetlands were water quality, vegetation and water surface. However, other researchers have stated that sometimes ASPT could be incompatible with the water condition (Guntharee 2003; Czeniawska-Kusza 2005) and habitat diversity was considered a very great factor. In general, some biological parameters showed significant weaknesses. They were not completely accurate in dis- tinguishing the differences between places and spatial periods (Nemati et al. 2009).

We were also unable to give an accurate comment based on just one year of monitoring because benthic macroinvertebrates have a life cycle of more than a year (about 1.5). Our results showed that BMWPs were compatible with the ASPT index. Other studies in wetlands and rivers have confirmed and suggested that there is a correlation between the ASPT and BMWP (Batty et al. 2005; Galbrand et al. 2007; Nemati et al. 2009). It should be noted that BMWPs have to be used for rivers (based on their sensitivity to organic materials). This may not be suitable for pollution monitoring of wetlands, but these indicators could provide us some useful information for water quality assessments in wetlands (Batty et al. 2008). Davis et al. (2006) wrote an article entitled "Can biological assessments of rivers be also used for wetlands?" They used variety of methods and indices in Australia and claimed that all these methods and indices were suitable for wetlands.

\section{The correlation between biological indices and water quality parameters}

Biological indicators had a compatible relationship with richness and the BMWP index at the level of $1 \%$ (Table 11). The relation between diversity indices was predictable because all of them were under the influence of species number. Also, their positive correlation with BMWP showed that with increasing diversity the BMWP index also increased. BMWP was also positively correlated with the indices of richness and Benthic macro invertebrate diversity, as well as ASPT (Table 11). These two indicators were compatible with each other and the ASPT could increase the accuracy of information provided by the BMWP. Hilsenhoff was unlike other indicators, so the correlation coefficient had a reverse trend compared to other indices and it even showed a negative correlation related to the ASPT and Simpson indices (Table 11). These differences were solely due to various scoring systems. In general, diversity and biological indicators confirmed the results obtained from the study by Hilsenhoff. Thus, the differences in the correlation coefficients were only in terms of number,

Table 11. Spearman correlation coefficients between diversity and biotic indices used in this study

\begin{tabular}{|l|c|c|c|c|c|c|c|}
\hline & Richness & Shannon & Simpson & Margalef & HBI & BMWP & ASPT \\
\hline Richness & 1 & & & & & \\
\hline Shannon & $0.461^{* *}$ & 1 & & & & \\
\hline Simpson & 0.188 & $0.925^{* *}$ & 1 & & & \\
\hline Margalef & $0.690^{* *}$ & $0.521^{* *}$ & $0.373^{* *}$ & 1 & & \\
\hline HBI & 0.156 & -0.172 & $-0.288^{* *}$ & -0.096 & 1 & & \\
\hline BMWP & $0.924^{* *}$ & $0.423^{* *}$ & 0.154 & $0.695^{* *}$ & 0.016 & 1 & \\
\hline ASPT & 0.055 & -0.001 & -0.083 & 0.143 & $-0.313^{* *}$ & $0.410^{* *}$ & 1 \\
\hline
\end{tabular}

${ }^{* *}$ Corelation is significant at the $99 \%$ level. 
but from the quality point of view, they confirmed each other. These results have been confirmed by other researchers utilizing these biomarkers for water quality assessments (Azrina et al. 2005; Batty et al. 2005; Galbrand et al. 2007; Taowu et al. 2008; Nemati et al. 2009).

The correlation between indicators of water quality parameters is shown in Table 12. None of the indices had a statistically significant correlation with nitrate and EC. The richness index showed a positive correlation with temperature, depth and ammonium. On the other hand, it had a negative correlation with Turbidity, TSS and TDS. In the study of Chawaka et al (2018) the total taxa richness of macroinvertebrates was positively correlated with dissolved oxygen, suggesting that conditions favoring a high dissolved oxygen concentration promote a higher macroinvertebrate richness. The Shannon-Wiener diversity index had a positive correlation with depth and ammonium, and a negative correlation with nitrite and hardness. In addition, the Simpson index had a positive correlation with depth, ammonium, $\mathrm{COD}$ and $\mathrm{BOD}_{5}$, and a negative correlation with nitrite and hardness. The Margalef index was positively correlated with temperature and depth, and negatively with nitrite, hardness, turbidity and TSS. Overall, the results indicated that Richness, Shannon, Simpson and Margalef indices could be influenced by environmental non-living factors. It was clear that depth had a significant correlation with all those indices. This could be justified by the shallowness of the pond located at the edge of the wetland, which was a result of various reasons such as water turbulence during storms, human activities such as fishing, sailing, etc. Thus, these factors could affect the bed wetland too. As a result, the presence of benthic animals was less in number. Not only could demographic factors have been affected by physicochemical parameters, they could also have had a direct impact on the growth of microbenthic organisms, as in nitrite, hardness, turbidity, $\mathrm{pH}$ (while being outside the range of tolerance of benthic organisms), TSS, TS, etc. They could also have had an indirect impact (only the numeric form), as in ammonium, which showed a positive correlation. On the one hand, there should have been a negative correlation as an unpleasant agent, on the other, we suspect that this could be a sign of organic decomposition as a result of better food conditions for some benthic invertebrates. Therefore, a positive correlation was shown. The indirect influence of water quality parameters on biological communities, such as the effect of nutrient on algae as a food source for animal organisms in other research, has also been considered (Angradi and Jicha 2010). Similar results have also been found in other research (Azrina et al. 2005; Batty et al. 2005; Sharma et al. 2006). HBI had a positive correlation with temperature and ammonium, and a negative correlation with nitrite and $\mathrm{pH}$. As mentioned previously, the increase in HBI meant more water pollution, since this was more sensitive to the increase of organic pollutants. Thus, when water pollution (mainly organic) increased, $\mathrm{BOD}_{5}$ and $\mathrm{COD}$ also increased. The same is true for HBI. As seen, there was a positive correlation between them. BMWP was positively correlated with temperature, depth, and ammonium, and negatively correlated with nitrite, phosphate, hardness and $\mathrm{pH}$. BMWP was influenced by a number of benthic families at each station. Hence the positive correlation between population indices and environmental parameters was also correct. The relationship between the above and some other parameters was reported in House and Whitley wetlands

Table 12. Spearman correlation coefficients between indices and water physicochemical variables

\begin{tabular}{|l|c|c|c|c|c|c|c|}
\hline & Richness & Shannon & Simpson & Margalef & HBI & BMWP & ASPT \\
\hline Temp. $\left[{ }^{\circ} \mathrm{C}\right]$ & $0.295^{* *}$ & -0.012 & -0.121 & $0.437^{* *}$ & $0.284^{*}$ & $0.244^{*}$ & -0.075 \\
\hline Depth $[\mathrm{m}]$ & $0.279^{*}$ & $0.388^{* *}$ & $0.378^{* *}$ & $0.249^{*}$ & 0.120 & $0.261^{*}$ & -0.058 \\
\hline $\mathrm{DO}\left[\mathrm{mg} \mathrm{dm}^{-3}\right]$ & 0.023 & 0.004 & -0.023 & 0.130 & -0.065 & 0.093 & $0.311^{* *}$ \\
\hline $\mathrm{PO}_{4}^{-}\left[\mathrm{mg} \mathrm{dm}^{-3}\right]$ & -0.119 & -0.208 & 0.073 & -0.174 & -0.181 & $-0.248^{*}$ & 0.189 \\
\hline $\mathrm{NO}_{3}^{-}\left[\mathrm{mg} \mathrm{dm}^{-3}\right]$ & -0.005 & -0.089 & -0.111 & -0.037 & 0.167 & 0 & 0.044 \\
\hline $\mathrm{NO}_{2}^{-}\left[\mathrm{mg} \mathrm{dm}^{-3}\right]$ & 0.149 & $-0.316^{* *}$ & $-0.229^{*}$ & $-0.382^{* *}$ & $-0.519^{* *}$ & $-0.604^{* *}$ & $-0.407^{* *}$ \\
\hline $\mathrm{NH}_{4}^{+}\left[\mathrm{mg} \mathrm{dm}^{-3}\right]$ & $0.271^{*}$ & $0.304^{* *}$ & $0.225^{*}$ & 0.209 & $0.285^{*}$ & $0.254^{*}$ & -0.041 \\
\hline${\text { Alkalinity }\left[\mathrm{mg} \mathrm{dm}^{-3}\right]}^{0.076}$ & -0.035 & -0.101 & -0.219 & 0.068 & 0.095 & -0.056 \\
\hline Hardness $\left[\mathrm{mg} \mathrm{dm}^{-3}\right]$ & 0.071 & $-0.285^{*}$ & $-0.372^{* *}$ & $-0.388^{* *}$ & 0.139 & $-0.245^{*}$ & $-0.343^{* *}$ \\
\hline Turbidity $\left[\mathrm{NTU}^{*}\right]$ & $-0.288^{* *}$ & -0.029 & -0.203 & $-0.302^{* *}$ & -0.030 & -0.055 & 0.137 \\
\hline EC $\left[\mu S \mathrm{~cm}^{-1}\right]$ & -0.011 & -0.040 & 0.127 & 0.167 & -0.060 & -0.032 & 0.014 \\
\hline pH & 0.059 & -0.049 & 0.109 & 0.097 & $-0.282^{*}$ & $-0.255^{*}$ & 0.073 \\
\hline TDS $\left[\mathrm{mg} \mathrm{dm}^{-3}\right]$ & $-0.224^{*}$ & -0.185 & 0.110 & 0.075 & 0.014 & -0.048 & -0.123 \\
\hline TSS $\left[\mathrm{mg} \mathrm{dm}^{-3}\right]$ & $-0.288^{* *}$ & -0.029 & -0.203 & $-0.302^{* *}$ & -0.030 & -0.055 & -0.137 \\
\hline BOD ${ }_{5}\left[\mathrm{mg} \mathrm{dm}^{-3}\right]$ & 0.034 & 0.022 & $0.284^{* *}$ & 0.054 & -0.158 & 0.052 & 0.017 \\
\hline COD $\left[\mathrm{mg} \mathrm{dm}^{-3}\right]$ & -0.002 & 0.020 & $0.400^{* *}$ & 0.054 & -0.138 & -0.031 & 0.029 \\
\hline
\end{tabular}

${ }^{\star}$ Correlation is significant at the $95 \%$ level, ${ }^{\star *}$ Correlation is significant at the $99 \%$ level 
(Batty et al. 2005). The ASPT index was positively correlated with dissolved oxygen and negatively with nitrite and hardness, as also claimed by other researchers (Nemati et al. 2009).

Individual organisms survive within specific ranges of temperature, water, and chemical conditions. It is believed that climate change and water quality (physical, chemical and biological conditions) play a decisive role in species composition in ecosystems, affecting their ecological structure, function and biodiversity (Jin et al. 2009). If organisms are exposed to unfavorable climatic conditions outside their normal environmental range and the health of their ecosystem is in decline, they must adapt or migrate, or they will perish. For example, worsening water quality will affect bird migration or nesting. In general, climate change and the decline of overall ecosystem health will have significant effects on the biodiversity of freshwater ecosystems, and it is very likely to have both direct and indirect consequences on the organisms and the structure and function of freshwater ecosystems (Desta et al. 2012; Zacchei et al. 2011). Wetlands are habitats and stopping surfaces for many bird species. According to Mitsch and Gosselink (2007), over $80 \%$ of migratory birds are dependent on wetlands as a stopping ground in their travels. However, water birds are also vulnerable to the impacts of climate change and water quality. Several aspects of climate change will affect not only wetlands, but also the water birds that use them in a number of different ways (Browne and Dell 2007). These changes can have effects on the timing and distance travelled during waterfowl migration (Browne and Dell 2007). Furthermore, climate variability can also affect populations and lead to changes in the range distribution of water birds their and abundance indirectly through trophic level impacts and water pollution on food availability (Butler and Taylor 2005). Therefore, as wetlands become affected and dried out as a result of climate change and water quality declines, aquatic birds will lose their stopping grounds. Bates et al. (2008) have also confirmed that the drying out of wetlands due to changes in climate will affect the migration success of birds that use them as stopovers.

\section{Conclusions}

The use of bio indicators (HBI, BMWP and ASPT), Margalef, Simpson and Shannon indices as well as richness at the level of family for water quality assessment in wetland was found to be effective such that their changes and water quality classifications showed reasonable consistency with all factors. The results generally showed that bioindicators like BMWP and ASPT were suitable to assess the water quality of the Choghakhor Wetland. Water in the studied areas was qualified as av- erage and severely polluted. However, we were unable to exploit the full potential of these indicators. Some other factors, such as the short time of study, life cycle and the presence of benthic macroinvertebrate, the use of family taxonomy, consistency, lack of accurate fundamental studies in this field and other ecological factors could also affect macroinvertebrate benthic structure. Ideally, the biological monitoring of water quality should have been conducted within at least a two-year period. Changes in the structure of the benthic invertebrate communities at different seasons and stations could be caused by environmental disturbances. The water quality of Choghakhor Wetland was evaluated to fall into the average and severe pollution class, so it seemed that all these indices showed a similar result. It can be argued that the migration of birds is at risk due to the declining of the overall health of the Choghakhor Wetland ecosystem.

\section{Acknowledgement}

The authors wish to thank the Department of Environmental Protection Agency of Chaharmahal Bakhtiari province. They also wish to thank Mr. Ebrahim Motaghi and Said Asadollah for their excellent technical assistance during the field work experiment.

\section{References}

Alvarez-Mieles G., Irvine K., Griensven AV., Arias-Hidalgo M., Torres A., Mynett A., 2013, Relationships between aquatic biotic communities and water quality in a tropical river-wetland system (Ecuador), Environ. Sci. Policy 34:115-137.

Angradi T.R., Jicha T.M., 2010, Mesohabitat-specific macroinvertebrate assemblage responses to water quality variation in mid-continent (North America) great rivers, Ecol. Indic. 10: 943-954.

[APHA] American Public Health Association, 1998, Standard Methods for the Examination of water and waste water, New York, 1496 pp.

Ayers R.S., Westcot D.W., 1985, Water quality for agriculture, FAO Irrigation and Drainage Paper 29 Rev. 1, FAO, Rome, 174 pp.

Azrina M.Z., Yap C.K., Rahim Ismail A., Ismail A., Tan S.G., 2005, Anthropogenic impacts on the distribution and biodiversity of benthic macroinvertebrates and water quality of the Langat River, Peninsular Malaysia, Ecotoxicol. Environ. Saf. 64(3): 337-347.

Barbour M.T., Gerritsen J., Geriffith G.E., Fridenborg R., McCarroon E., White J.S., Bastian M.L., 1996, A framework for biological criteria for Florida streams using benthic macroinvertebrates, J. N. Amer. Benthol. Soc. 15(2): 185211.

Barbour M.T., Gerritsen J., Snyder B.D., Stribling J.B., 1999, Rapid bioassessment protocols for use in streams and 
wadeable rivers: Periphyton, benthic macroinvertebrates, and fish, US EPA, Washington, 337 pp.

Bates B.C., Kundzewicz Z.W., Wu S., Palutikof J.P., 2008, Climate change and water, Technical Paper of the Intergovernmental Panel on Climate Change, IPCC Secretariat, Geneva, 214 pp.

Batty L.C., Atkin L., Manning D.A.C., 2005, Assessment of the ecological potential of mine-water treatment wetlands using a baseline survey of macroinvertebrate communities, Environ. Pollut. 138(3): 412-419.

Batzer D.P., Rader R.B., Wissinger S.A., 1999, Invertebrates in freshwater wetlands of North America: Ecology and management, Wiley, New York, 1120 pp.

Bode R.W., Novak M.A., Abele L.E., 1996, Quality assurance work plan for biological stream monitoring in New York State, NYS Dept. of Environmental Conservation, Albany, $92 \mathrm{pp}$.

Browne D.M., Dell R. (eds), 2007, Conserving waterfowl and wetlands amid climate change, Ducks Unlimited Inc, Memphis, 50 pp.

Burton T.M., Uzarski D.G., Gathman J.P., Genet J.A., Keas B.A., Stricker C.A., 1999, Development of a preliminaryinvertebrate index of biotic integrity for Lake Huron coastal wetlands, Wetlands 19(4): 869-882.

Butler R.W., Taylor W., 2005, A Review of climate change impacts on birds. USDA Forest Service Gen. Tech. Rep. PSW-GTR-191: 1107-1109.

Cabecinha E., Silva-Santos P., Cortes R., Cabral J.A., 2007, Applying a stochastic-dynamic methodology (StDM) to facilitate ecological monitoring of running waters, using selected trophic and taxonomic metrics as state variables, Ecol. Modell. 207: 109-127.

Chawaka S.N., Boets P., Mereta S.T.T., Ho L.L.M., Goethals P., 2018, Using macroinvertebrates and birds to assess the environmental status of wetlands across different climatic zones in Southwestern Ethiopia, Wetlands 38(4): 653-665.

Czeniawska-Kusza I., 2005, Comparing modified Biological Monitoring Working Party score system and several biological indices based on macroinvertebrates for water quailty assessment, Limnologica 35(3): 169-176.

Davis J., Horwitz P., Norris R., Chessman B., McGuire M., Sommer B., 2006, Are river bioassessment methods using macroinvertebrates applicable to wetlands, Hydrobiologia 572(1): 115-128.

Desta H., Lemma B., Fetene A., 2010, Aspects of climate change and its associated impacts on wetland ecosystem functions, J. Am. Sci. 8(10): 582-596.

Hettiarchchi M., Anurangi J., De Alwis A., 2011, Characterisation and description of surface water quality in the threatened urban wetlands around the City of Colombo, Wetl. Ecol. Manag. 5: 10-19.

Fishar M.R., Williams W.P., 2008, The development of a biotic pollution index for the river Nile in Egypt, Hydrobiologia 598(1): 17-34.
Galbrand C., Lemieux I.G., Ghaly A.E., Cote R., Verma M., 2007, Assessment of constructed wetland biological integrity using aquatic macroinvertebrates, J. Biol. Sci. 7(2): 52-65.

Gencer T., Nilgun K., 2010, Applications of various diversity indices to benthic macroinvertebrate assemblages in streams of a natural park in Turkey, Rev. Hydrobiol. 3(2): 111-125.

Getachew M, Ambelu A., Tiku S., Legesse W., Adugna A., Kloos H., 2012, Ecological assessment of Cheffa Wetland in the Borkena Valley, northeast Ethiopia: Macroinvertebrate and bird communities, Ecol. Indic. 15(1): 63-71.

Guntharee S., 2003, Benthic mcroinvertebrates as a biological index of water quality in the Lower Thachin River, Silpakorn University Int. J. 3: 168-183.

Harrington F.A., 1977, A guide to the mammals of Iran, Fardin Press, Tehran, 89 pp.

Hawkes H.A., 1997, Tacnical note: Origin and development of the Biological Monitoring Working Party score system, Water. Res. 32: 964-968.

Gernes M.C, Helgen J.C, 2002, Indexes of Biological Integrity (IBI) for large depressional wetlands in Minnesota, Minnesota Pollution Control Agency, St. Paul, 86 pp.

Hilsenhoff W.L., 1988, Rapid field assessment for organic pollution with a family-level biotic index, J. N. Amer. Benthol. Soc. 7(1): 65-68.

Hsu C.B., Hsieh H.L., Yang L., Wu S.H., Chang J.S., Hsiao S.C., Su H.C., Yeh C.H., Ho Y.S., Lin H.J., 2011, Biodiversity of constructed wetlands for wastewater treatment, Ecol. Eng. 37(10): 1533-1545.

Hynes H.B.N., 1977, A key to adult and nymphs of the British stoneflies (Plecoptera) with notes on the ecology and distribution, Freshwater Biological Association, Ambleside, $92 \mathrm{pp}$.

Jin C., Cant B., Todd C., 2009, Climate change impacts on wetlands in Victoria and implications for research and policy, Arthur Rylah Institute for Environmental Research Technical Series No. 199, Heidelberg, 49 pp.

Krebs C.J., 2001, Ecology: The experimental analysis of distribution and abundance, Benjamin Cummings, San Francisco, 695 pp.

Li X., Manman C., Anderson B.C., 2009, Design and performance of a water quality treatment wetland in a public park in Shanghai, China, Ecol Eng. 35(1): 18-24.

Mandaville S.M., 2002, Benthic macroinvertebrates in freshwater - Taxa tolerance values, metrics and protocols (Project H-1), Soil \& Water Conservation Society of Metro Halifax, Mandaville, $128 \mathrm{pp}$.

Mansoori J., 2008, A guide to the birds of Iran, Farzaneh, Tehran, 513 pp (in Persian, English summary).

Mereta S.T., Boets P., Meester L.D., Goethals P., 2013, Development of a multimetric index based on benthic macroinvertebrates for the assessment of natural wetlands in Southwest Ethiopia, Ecol. Indic. 29: 510-521.

Milligan M.R., 1997, Identification manual of the aquatic oligochaeta of Florida. Vol I: Freshwater Oligochaetes, State 
of Florida - Dept. of Environmental Protection, Tallahassee, $187 \mathrm{pp}$.

Mitsch W.J, Gosselink J.G., 2007, Wetlands, John Wiley and Sons, Hoboken, 582 pp.

Nemati M., Ebrahimi E., Mirghafari N., Safianian A., 2009, Biological assessment of the Zayandeh Rud River, Iran, using benthic macroinvertebrate, Limnologica 40(3): 1-6.

Pescador M.L., Rasmussen A.K., Harris S.C., 2004, Identification manual for the caddisfly (Trichoptera) larvae of Florida, State of Florida - Dept. of Environmental Protection, Tallahassee, $141 \mathrm{pp}$.

Pinel-Alloul B., Methot G., Lapierre L., Willsie A., 1995, Macroinvertebrate community as a biological indicator of ecological and toxicological factors in Lake Saint-Francois (Quebec), Environ. Pollut. 91(1): 65-87.

Rader R.B., Batzer D.P., Wissinger S.A., 2001, Bioassessment and management of North American freshwater wetlands, John Wiley and Sons, New York, 480 pp.

Ramesh C.S., Jitendra S.R., 2009, Monitoring of aquatic macroinvertebrates as bioindicator for assessing the health of wetlands, Ecol. Indic. 9(1): 118-128.

[RCW] Ramsar Convention on Wetlands, 2002, Background papers on wetland values and functions: Reservoirs of biodiversity, Ramsar Convention Bureau, Gland, 168 pp.

Rosenberg D.M., Resh V.H. (eds), 1993, Freshwater biomonitoring and benthic macroinvertebrates. Chapman \& Hall, New York, 488 pp.

Rosenberg D.M., Davies I.J., Cobb D.G., Wiens A.P., 1999, Protocols for maesuring biodiversity: Benthic macroinvertebrates in freshwaters, Freshwater Institute, Winnipeg, 43 pp.

Sharma M.P., Sharma S., Geol V., Sharma P., Kumar A., 2006, Water quality assessment of Behta River using benthic macroinvertebrates, Life. Sci. 3(1): 68-74.

Shivandi D., Nazarian A., Davoodi G.H., Riahi M., 1999, Environment aspect in Chahar Mahal Bakhtiari Province, Society of Efset Edition and Emission, Shahre Kord, 121 pp.

Taylor B.R., Baily R.C., 1997., Technical evaluation on methods for benthic invertebrates data analysis and interpretation. Final Report, Canada Centre for Mineral and Energy Technology, Ottawa, 90 pp.
Taowu M., Zijian W., Qinghui H., Hai W., Chunxia W., Shengbiao H., 2008, Selection of benthic macroinvertebrate-based multimetrics and preliminary establishment of biocriteria for the bioassessment of the water quality of Taihu Lake, China, Acta Ecol. Sin. 28(3): 1192-1200.

Tiner R.W., 1999, Wetland indicators: A guide to wetland identification, delineation, classification, and mapping, CRC Press, Boca Raton, 606 pp.

[USEPA] United States Environmental Protection Agency, 2000, Ambient aquatic life criteria for dissolved oxygen (saltwater): Cape Cod to Cape Hatteras, Office of Water, Office of Science and Technology, Washington.

Walen J.K., 2002, Assessment of stream habitat, fish, macroinvertebrates, sediment and water chemistry for eleven streams in land between the Lakes National Recreation Area, Kentucky and Tennessee, United States Dept of Agriculture Forest Service, Blacksburg, $71 \mathrm{pp}$.

Wally W.J., Hawkes H.A., 1996, A computer-based reappraisal of the Biological Monitoring Working Party score system using data from the 1990 river quality survey of England and Wales, Water Res. 30(9): 2086-2094.

Wally W.J., Hawkes H.A., 1997, A computer-based development of the Biological Monitoring Working Party score system incorporating abundance rating, site type and indicatore value, Water Res. 31(2): 201-210.

Washington H.G., 1984, Diversity, biotic and similarity indices. A review with special relevance to aquatic ecosystems, Water Res. 18(6): 653-694.

Wilhm J.L., Dorris T.C., 1968, Biological parameters for quality criteria, Bioscience 18(6): 477-481.

Yimer H.D., Mengistou S., 2009, Water quality parameters and macroinvertebrates index of biotic integrity of the Jimma Wetlands, Southwestern Ethiopia, J. Wetlands Ecol. 3: 77-93.

Zar J.H., 1999, Biostatistical analysis, Prentice-Hall, Upper Saddle River, 663 pp.

Zacchei D., Battisti C., Carpaneto G.M., 2011, Contrasting effects of water stress on wetland-obligated birds in a semi-natural Mediterranean wetland, Lakes Reserv. Res. Manag. 16(4): 281-286. 